\title{
ON THE WELL-POSEDNESS OF THE HALL-MAGNETOHYDRODYNAMICS SYSTEM IN CRITICAL SPACES
}

\author{
RAPHAËL DANCHIN AND JIN TAN
}

\begin{abstract}
We investigate the existence and uniqueness issues of the 3D incompressible Hall-magnetohydrodynamic system supplemented with initial velocity $u_{0}$ and magnetic field $B_{0}$ in critical regularity spaces.

In the case where $u_{0}, B_{0}$ and the current $J_{0}:=\nabla \times B_{0}$ belong to the homogeneous Besov space $\dot{B}_{p, 1}^{\frac{3}{p}-1}, 1 \leq p<\infty$, and are small enough, we establish a global result and the conservation of higher regularity. If the viscosity is equal to the magnetic resistivity, then we obtain the global well-posedness provided $u_{0}, B_{0}$ and $J_{0}$ are small enough in the larger Besov space $\dot{B}_{2, r}^{\frac{1}{2}}, r \geq 1$. If $r=1$, then we also establish the local existence for large data, and exhibit continuation criteria for solutions with critical regularity.

Our results rely on an extended formulation of the Hall-MHD system, that has some similarities with the incompressible Navier-Stokes equations.
\end{abstract}

\section{INTRODUCTION}

We are concerned with the following three dimensional incompressible resistive and viscous Hall-magnetohydrodynamics system (Hall-MHD):

$$
\begin{aligned}
& \partial_{t} u+\operatorname{div}(u \otimes u)+\nabla P=(\nabla \times B) \times B+\mu \Delta u, \\
& \operatorname{div} u=0, \\
& \partial_{t} B=\nabla \times((u-h \nabla \times B) \times B)+\nu \Delta B,
\end{aligned}
$$

supplemented with the initial conditions

$$
(u(0, x), B(0, x))=\left(u_{0}(x), B_{0}(x)\right), \quad x \in \mathbb{R}^{3} .
$$

The unknown vector-fields $u=u(t, x)$ and $B=B(t, x)$, and scalar function $P=$ $P(t, x)$ with $t \geq 0$ and $x \in \mathbb{R}^{3}$ represent the velocity field, the magnetic field and the scalar pressure, respectively. The parameters $\mu$ and $\nu$ are the fluid viscosity and the magnetic resistivity, while the dimensionless number $h$ measures the magnitude of the Hall effect compared to the typical length scale of the fluid. In accordance with (1.2), we assume that $\operatorname{div} u_{0}=0$ and, for physical consistency, since a magnetic field has to be divergence free, we suppose that $\operatorname{div} B_{0}=0$, too, a property that is conserved through the evolution.

The above system is used to model the evolution of electrically conducting fluids such as plasmas or electrolytes (then, $u$ represents the ion velocity), and takes into account the fact that in a moving conductive fluid, the magnetic field can induce

2010 Mathematics Subject Classification. 35Q35; 76D03; 86A10.

Key words and phrases. Magnetohydrodynamics, Hall effect, Well-posedness, critical regularity. 
currents which, in turn, polarize the fluid and change the magnetic field. That phenomenon which is neglected in the classical MHD equations, is represented by the Hall electric field $E_{H}:=h J \times B$ where the current $J$ is defined by $J:=$ $\nabla \times B$. Hall term plays an important role in magnetic reconnection, as observed in e.g. plasmas, star formation, solar flares, neutron stars or geo-dynamo (for more explanation on the physical background of the Hall-MHD system, one can refer to 17, 4, 13, 14, 18, 19]).

Despite its physical relevance, the Hall-MHD system has been considered only rather recently in mathematics, following the work by Acheritogaray, Degond, Frouvelle and Liu in [1] where the Hall-MHD system was formally derived both from a two fluids system and from a kinetic model. Then, in [8, Chae, Degond and Liu showed the global existence of weak solutions as well as the local well-posedness for initial data $u_{0}$ and $B_{0}$ in Sobolev spaces $H^{s}$ with $s>5 / 2$. Weak solutions have been further investigated by Dumas and Sueur in [12] both for the Maxwell-LandauLifshitz system and for the Hall-MHD system. In [9, 25, Serrin type continuation criteria for smooth solutions and the global existence of strong solutions emanating from small initial data have been obtained. In [2, it has been observed that the possible blow-up of smooth solutions may be controlled in terms of the velocity only. More well-posedness results of strong solutions for less regular data in Sobolev or Besov spaces, have been established in [5, 20] and 21, and the convergence to the MHD system with no Hall-term for $h \rightarrow 0$ has been addressed in 24]. Examples of smooth data with arbitrarily large $L^{\infty}$ norms giving rise to global unique solutions have been exhibited very recently in [15.

Our main goal here is to establish the well-posedness of the Hall-MHD system with initial data in critical spaces. In contrast with the classical MHD system (that corresponds to $h=0$ ) however, the system under consideration does not have any scaling invariance owing to the coexistence of the Hall term in (1.3) and of the Lorentz force in (1.1), and we have to explain what we mean by critical regularity.

On the one hand, for $B \equiv 0$, the Hall-MHD system reduces to the incompressible Navier-Stokes:

$$
\left\{\begin{array}{l}
\partial_{t} u+(u \cdot \nabla) u+\nabla P=\mu \Delta u, \\
\operatorname{div} u=0, \\
\left.u\right|_{t=0}=u_{0},
\end{array}\right.
$$

which are invariant for all $\lambda>0$ by the rescaling

$$
u(t, x) \leadsto \lambda u\left(\lambda^{2} t, \lambda x\right) \text { and } P(t, x) \leadsto \lambda^{2} P\left(\lambda^{2} t, \lambda x\right)
$$

provided the initial velocity $u_{0}$ is rescaled according to

$$
u_{0}(x) \leadsto \lambda u_{0}(\lambda x) .
$$

On the other hand, if the fluid velocity in (1.3) is 0, then we get the following Hall equation for $B$ :

$$
\left\{\begin{array}{l}
\partial_{t} B+h \nabla \times((\nabla \times B) \times B)=\nu \Delta B, \\
\left.B\right|_{t=0}=B_{0},
\end{array}\right.
$$

which is invariant by the rescaling

$$
B(t, x) \sim B\left(\lambda^{2} t, \lambda x\right)
$$


provided the data $B_{0}$ is rescaled according to

$$
B_{0}(x) \sim B_{0}(\lambda x) .
$$

Therefore, if $h>0$ and if we neglect the Lorentz force in (1.1), then it is natural to work at the same level of regularity for $u$ and $\nabla B$, while for $h=0$ (the classical MHD system) $u$ and $B$ have the same scaling invariance.

The way to reconcile the two viewpoints is to look at the current function $J=$ $\nabla \times B$ as an additional unknown. Now, owing to the vector identity

$$
\nabla \times(\nabla \times v)+\Delta v=\nabla \operatorname{div} v
$$

and since $B$ is divergence free, we have $\Delta B=-\nabla \times J$, whence

$$
B=\operatorname{curl}^{-1} J:=(-\Delta)^{-1} \nabla \times J,
$$

where the -1 order homogeneous Fourier multiplier $\mathrm{curl}^{-1}$ is defined on the Fourier side by

$$
\mathcal{F}\left(\operatorname{curl}^{-1} J\right)(\xi):=\frac{i \xi \times \widehat{J}(\xi)}{|\xi|^{2}} .
$$

With that notation, one gets the following extended Hall-MHD system:

$$
\left\{\begin{array}{l}
\partial_{t} u+\operatorname{div}(u \otimes u)-\mu \Delta u+\nabla P=(\nabla \times B) \times B \\
\operatorname{div} u=0 \\
\partial_{t} B-\nabla \times((u-h J) \times B)-\nu \Delta B=0, \\
\partial_{t} J-\nabla \times\left(\nabla \times\left((u-h J) \times \operatorname{curl}^{-1} J\right)\right)-\nu \Delta J=0 .
\end{array}\right.
$$

In contrast with the original Hall-MHD system, the above extended system has a scaling invariance (the same as for the incompressible Navier-Stokes equations). Studying whether the Hall-MHD system written in terms of $(u, B, J)$ is well-posed in the same functional spaces as the velocity in $(N S)$, and if similar blow-up criteria and qualitative behavior may be established is the main aim of the paper.

Notation. We end this introductory part presenting a few notations. We denote by $C$ harmless positive 'constants' that may change from one line to the other, and we sometimes write $A \lesssim B$ instead of $A \leq C B$. Likewise, $A \sim B$ means that $C_{1} B \leq A \leq C_{2} B$ with absolute constants $C_{1}, C_{2}$. For $X$ a Banach space, $p \in[1, \infty]$ and $T \in(0, \infty]$, the notation $L^{p}(0, T ; X)$ or $L_{T}^{p}(X)$ designates the set of measurable functions $f:[0, T] \rightarrow X$ with $t \mapsto\|f(t)\|_{X}$ in $L^{p}(0, T)$, endowed with the norm $\|\cdot\|_{L_{T}^{p}(X)}:=\|\| \cdot\left\|_{X}\right\|_{L^{p}(0, T)}$. For any interval $I$ of $\mathbb{R}$, we agree that $\mathcal{C}(I ; X)$ (resp. $\left.\mathcal{C}_{b}(I ; X)\right)$ denotes the set of continuous (resp. continuous and bounded) functions from $I$ to $X$. We keep the same notation for functions with several components.

Acknowledgments. The authors are indebted to the anonymous referees for their wise suggestions that contributed to improve the final version of this work. The first author is partially supported by the ANR project INFAMIE (ANR-15-CE400011). The second author has been partly funded by the Bézout Labex, funded by ANR, reference ANR-10-LABX-58. 


\section{MAIN RESUlts}

It is by now classical that the incompressible Navier-Stokes equations are wellposed (locally for large data or globally for small data) in all homogeneous Besov spaces $\dot{B}_{p, r}^{\frac{3}{p}-1}$ with $1 \leq p<\infty$ and $1 \leq r \leq \infty$, see [7, 10. Similar results have been obtained for the standard incompressible MHD system (that is with no Hall term) by C. Miao and B. Yuan in [16]. According to the scaling considerations of the above paragraph, it is natural to look for similar results for the Hall-MHD system written in its extended formulation (1.11).

Let us first consider the case of positive general coefficients $\mu, \nu$ and $h$, for data $\left(u_{0}, B_{0}, J_{0}\right)$ in $\dot{B}_{p, 1}^{\frac{3}{p}-1}$. Following the paper [10] by J.-Y. Chemin dedicated to the incompressible Navier-Stokes equations, we introduce for $T>0$, the space

$$
E_{p}(T):=\left\{z \in \mathcal{C}\left([0, T], \dot{B}_{p, 1}^{\frac{3}{p}-1}\right), \nabla_{x}^{2} z \in L^{1}\left(0, T ; \dot{B}_{p, 1}^{\frac{3}{p}-1}\right) \quad \text { and } \quad \operatorname{div}_{x} z=0\right\}
$$

and its global version $E_{p}\left(\right.$ with $\left.z \in \mathcal{C}_{b}\left(\mathbb{R}_{+} ; \dot{B}_{p, 1}^{\frac{3}{p}-1}\right)\right)$ if $T=+\infty$.

Our first result states the global well-posedness of the Hall-MHD system for small data in $\dot{B}_{p, 1}^{\frac{3}{p}-1}$, and conservation of higher order Sobolev regularity for any positive coefficients $\mu, \nu$ and $h$.

Theorem 2.1. Let $1 \leq p<\infty$ and $\left(u_{0}, B_{0}\right) \in \dot{B}_{p, 1}^{\frac{3}{p}-1}$ with $\operatorname{div} u_{0}=\operatorname{div} B_{0}=0$ and $J_{0}:=\nabla \times B_{0} \in \dot{B}_{p, 1}^{\frac{3}{p}-1}$. There exists a constant $c>0$ depending only on $p$ and $\mu / \nu$ such that, if

$$
\left\|u_{0}\right\|_{\dot{B}_{p, 1}^{\frac{3}{p}-1}}+\left\|B_{0}\right\|_{\dot{B}_{p, 1}^{\frac{3}{p}-1}}+h\left\|J_{0}\right\|_{\dot{B}_{p, 1}^{\frac{3}{p}-1}}<c \mu,
$$

then the Cauchy problem (1.1)-(1.4) has a unique global solution $(u, B) \in E_{p}$, with $J:=\nabla \times B \in E_{p}$. Furthermore,

$$
\|u\|_{E_{p}}+\|B\|_{E_{p}}+h\|J\|_{E_{p}}<2 c \mu .
$$

If, in addition, $u_{0} \in H^{s}$ and $B_{0} \in H^{r}$ with

$$
\frac{3}{p}-1<s \leq r \quad \text { and } \quad \frac{3}{p}<r \leq 1+s,
$$

then $(u, B) \in \mathcal{C}_{b}\left(\mathbb{R}_{+} ; H^{s} \times H^{r}\right), \nabla u \in L^{2}\left(\mathbb{R}_{+} ; H^{s}\right)$ and $\nabla B \in L^{2}\left(\mathbb{R}_{+} ; H^{r}\right)$ and the following energy balance is fulfilled for all $t \geq 0$ :

$$
\|u(t)\|_{L^{2}}^{2}+\|B(t)\|_{L^{2}}^{2}+2 \int_{0}^{t}\left(\mu\|\nabla u\|_{L^{2}}^{2}+\nu\|\nabla B\|_{L^{2}}^{2}\right) d \tau=\left\|u_{0}\right\|_{L^{2}}^{2}+\left\|B_{0}\right\|_{L^{2}}^{2} .
$$

Finally, in the case where only $J_{0}$ fulfills (2.1), there exists some time $T>0$ such that (1.1)-(1.4) has a unique local-in-time solution on $[0, T]$ with $(u, B, J)$ in $E_{p}(T)$, and additional Sobolev regularity is preserved.

Remark 1. For $h$ going to 0 , we recover the same smallness condition as in the paper [16] dedicated to the MHD system with no Hall-term.

Remark 2. Whether the smallness condition on $J_{0}$ may be omitted in the context of general critical regularity spaces $\dot{B}_{p, 1}^{\frac{3}{p}-1}$ is an open question. The difficulty not only comes from Hall term but also from the coupling between $u$ and $B$ through the term $\nabla \times(u \times B)$. For essentially the same reason, we do not know how to solve the system in $\dot{B}_{p, r}^{\frac{3}{p}-1}$ if $r>1$, unless $p=2$ and $\mu=\nu$, as we shall see later on. 
The key to the proof of Theorem 2.1 is to consider the extended Hall-MHD system (1.11), suitably rewritten in the form of a generalized Navier-Stokes system that may be solved by implementing the classical fixed point theorem in the Banach space $E_{p}$. In order to derive an appropriate formulation of the system, we need to use some algebraic identities. The first one is that for any couple of $C^{1}$ divergence free vector-fields $v$ and $w$ on $\mathbb{R}^{3}$, we have

$$
w \cdot \nabla v=\operatorname{div}(v \otimes w), \quad \text { where }(\operatorname{div}(v \otimes w))^{j}:=\sum_{k=1}^{3} \partial_{k}\left(v^{j} w^{k}\right) .
$$

Observe also that

$$
(\nabla \times w) \times w=(w \cdot \nabla) w-\nabla\left(\frac{|w|^{2}}{2}\right) .
$$

Hence, setting $Q:=P+|B|^{2} / 2$, equation (1.1) recasts in

$$
\partial_{t} u+\operatorname{div}(u \otimes u)+\nabla Q=\operatorname{div}(B \otimes B)+\mu \Delta u .
$$

After projecting (2.7) onto the set of divergence free vector fields by means of the Leray projector $\mathcal{P}:=\operatorname{Id}-\nabla(-\Delta)^{-1} \operatorname{div}$, we get

$$
\partial_{t} u-\mu \Delta u=Q_{a}(B, B)-Q_{a}(u, u),
$$

where the bilinear form $Q_{a}$ is defined by

$$
Q_{a}(v, w):=\frac{1}{2} \mathcal{P}(\operatorname{div}(v \otimes w)+\operatorname{div}(w \otimes v)) .
$$

Next, by using the identity

$$
\nabla \times(w \times v)=v \cdot \nabla w-w \cdot \nabla v,
$$

one can rewrite Hall term as

$$
\nabla \times(J \times B)=B \cdot \nabla J-J \cdot \nabla B .
$$

Hence, combining with (2.5), equation (1.3) recasts in

$$
\partial_{t} B-\nu \Delta B=Q_{b}(B, h J-u),
$$

where

$$
Q_{b}(v, w):=\operatorname{div}(v \otimes w)-\operatorname{div}(w \otimes v)=w \cdot \nabla v-v \cdot \nabla w,
$$

and the equation for $J$ may thus be written

$$
\partial_{t} J-h \Delta J=\nabla \times Q_{b}\left(\operatorname{curl}^{-1} J, h J-u\right) .
$$

Altogether, we conclude that the extended Hall-MHD system (1.11) recasts in

$$
\left\{\begin{array}{l}
\partial_{t} u-\mu \Delta u=Q_{a}(B, B)-Q_{a}(u, u), \\
\partial_{t} B-\nu \Delta B=Q_{b}(B, h J-u), \\
\partial_{t} J-\nu \Delta J=\nabla \times Q_{b}\left(\operatorname{curl}^{-1} J, h J-u\right), \\
(u(0, x), B(0, x), J(0, x))=\left(u_{0}, B_{0}, J_{0}\right) .
\end{array}\right.
$$

Set $U:=\left(U_{1}, U_{2}, U_{3}\right)$ with $U_{1}:=u, U_{2}:=B$ and $U_{3}:=J$. Then, (2.10) may be shortened into:

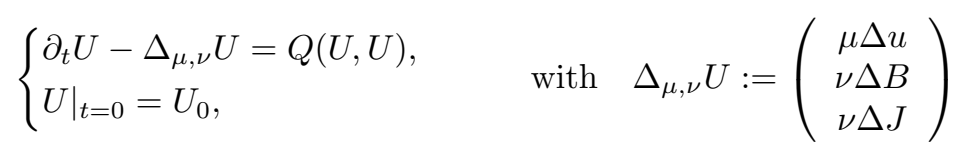


and where $Q: \mathbb{R}^{3} \times \mathbb{R}^{3} \rightarrow \mathbb{R}^{9}$ is defined by

$$
Q(V, W):=\left(\begin{array}{c}
Q_{a}\left(V_{2}, W_{2}\right)-Q_{a}\left(V_{1}, W_{1}\right) \\
Q_{b}\left(V_{2}, h W_{3}-W_{1}\right) \\
\nabla \times Q_{b}\left(\operatorname{curl}^{-1} V_{3}, h W_{3}-W_{1}\right)
\end{array}\right) .
$$

The gain of considering the above extended system rather than the initial one is that it is semi-linear, while the Hall-MHD system for $(u, B)$ is quasi-linear. The quadratic terms in the first two lines of (2.11) are essentially the same as for the incompressible Navier-Stokes equation. Owing to the Hall-MHD term in the last line however, one has to go beyond the theory of the generalized Navier-Stokes equations as presented in e.g. [3. Chap. 5] since the differentiation is outside instead of being inside the first variable of $Q_{b}$ (this actually prevents us from considering large $J_{0}$ 's and to handle regularity in Besov spaces $\dot{B}_{p, r}^{\frac{3}{p}-1}$ with $r>1$ ).

In the case1 $\mu=\nu$, there is a cancellation property that eliminates the Hall term when performing an energy method, so that one can obtain better results. In particular, one can prove the local well-posedness for general large data in $\dot{B}_{2,1}^{\frac{1}{2}}$ and the global well-posedness for small data in all spaces $\dot{B}_{2, r}^{\frac{1}{2}}$ with $r \in[1, \infty]$.

In order to explain where that cancellation comes from, let us introduce the function $v:=u-h J$ (that may be interpreted as the velocity of an electron, see 17. page 5). Recall the following vector identities:

$$
\nabla(w \cdot z)=(\nabla w)^{T} z+(\nabla z)^{T} w \quad \text { and } \quad\left(\nabla w-(\nabla w)^{T}\right) z=(\nabla \times w) \times z
$$

where $(\nabla w)_{i j}:=\partial_{j} w^{i}$ for $1 \leq i, j \leq 3$.

Hence, combining with (2.9) yields

$$
\begin{aligned}
\nabla \times(w \times z) & =z \cdot \nabla w-w \cdot \nabla z \\
& =\left(\nabla w-(\nabla w)^{T}\right) z+\left(\nabla z-(\nabla z)^{T}\right) w-2 w \cdot \nabla z+\nabla(w \cdot z) \\
& =(\nabla \times w) \times z+(\nabla \times z) \times w-2 w \cdot \nabla z+\nabla(w \cdot z) .
\end{aligned}
$$

Then, applying Identity (2.13) to the term $\nabla \times(v \times B)$, equation (1.3) turns into

$$
\partial_{t} B-\mu \Delta B=(\nabla \times v) \times B+J \times u-2 v \cdot \nabla B+\nabla(v \cdot B) .
$$

Taking $h \cdot$ curl of the above equation, and subtracting it from (2.7), we get

$$
\begin{aligned}
\partial_{t} v-\mu \Delta v=B \cdot \nabla B-u \cdot \nabla u-h \nabla \times & ((\nabla \times v) \times B) \\
& +h \nabla \times(u \times J)+2 h \nabla \times(v \cdot \nabla B)-\nabla Q .
\end{aligned}
$$

Therefore, since $h J=v-u$, in terms of unknowns $(u, B, v)$, the extended Hall-MHD system reads

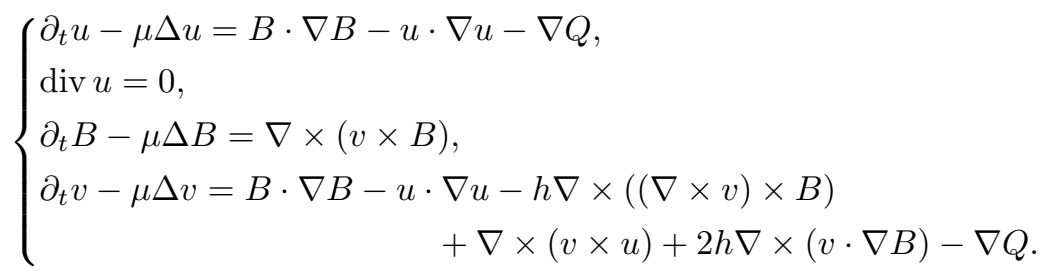

\footnotetext{
${ }^{1}$ That is made in most mathematical papers devoted to the Hall-MHD system even though it is not physically motivated.
} 
The only quasilinear term cancels out when performing an energy method, since

$$
(\nabla \times((\nabla \times v) \times B), v)_{L^{2}}=0 .
$$

After localization of the system by means of the Littlewood-Paley spectral cut-off operators $\dot{\Delta}_{j}$ defined in the Appendix, the above identity still holds, up to some lower order commutator term. This will enable us to prove the following local well-posedness result for large data in the critical Besov space $\dot{B}_{2,1}^{\frac{1}{2}}$, together with blow-up criteria involving critical norms.

Theorem 2.2. Assume that $\mu=\nu$. For any initial data $\left(u_{0}, B_{0}\right)$ in $\dot{B}_{2,1}^{\frac{1}{2}}$ with $\operatorname{div} u_{0}=\operatorname{div} B_{0}=0$ and $J_{0}:=\nabla \times B_{0} \in \dot{B}_{2,1}^{\frac{1}{2}}$, there exists a positive time $T$ such that the Cauchy problem (1.1)-(1.4) has a unique solution $(u, B) \in E_{2}(T)$ with $J:=\nabla \times B \in E_{2}(T)$. Moreover, if the maximal time of existence $T^{*}$ of that solution is finite, then

$$
\begin{aligned}
& \int_{0}^{T^{*}}\|(u, B, \nabla B)(t)\|_{L^{\infty}}^{2} d t=\infty \\
& \int_{0}^{T^{*}}\|(u, B, \nabla B)(t)\|_{\dot{B}_{2,1}^{\frac{5}{2}}} d t=\infty
\end{aligned}
$$

and, for any $\rho \in(2, \infty)$,

$$
\int_{0}^{T^{*}}\|(u, B, \nabla B)(t)\|_{\dot{B}_{\infty, \infty}^{\frac{2}{\rho}-1}}^{\rho} d t=\infty .
$$

Still for $\mu=\nu$, one can prove well-posedness in any critical space $\dot{B}_{2, r}^{\frac{1}{2}}$ with $r \in[1, \infty]$. Then, the components of the solution will belong to the following spaced:

$$
E_{2, r}(T):=\left\{v \in \widetilde{\mathcal{C}}_{T}\left(\dot{B}_{2, r}^{\frac{1}{2}}\right), \nabla^{2} v \in \widetilde{L}_{T}^{1}\left(\dot{B}_{2, r}^{\frac{1}{2}}\right) \quad \text { and } \operatorname{div} v=0\right\},
$$

where the letter $T$ is omitted if the time interval is $\mathbb{R}_{+}$.

Theorem 2.3. Assume that $\mu=\nu$. Consider initial data $\left(u_{0}, B_{0}\right)$ in $\dot{B}_{2, r}^{\frac{1}{2}}$ with $\operatorname{div} u_{0}=\operatorname{div} B_{0}=0$ and $J_{0}:=\nabla \times B_{0} \in \dot{B}_{2, r}^{\frac{1}{2}}$ for some $r \in[1, \infty]$. Then, the following results hold true:

(1) there exists a universal positive constant $c$ such that, if

$$
\left\|u_{0}\right\|_{\dot{B}_{2, r}^{\frac{1}{2}}}+\left\|B_{0}\right\|_{\dot{B}_{2, r}^{\frac{1}{2}}}+\left\|u_{0}-h J_{0}\right\|_{\dot{B}_{2, r}^{\frac{1}{2}}}<c \mu,
$$

then the Hall-MHD system has a unique global solution $(u, B)$ with $(u, B, J)$ in $E_{2, r}$.

(2) If only $\left\|u_{0}-h J_{0}\right\|_{\dot{B}_{2, r}^{\frac{1}{2}}}<c \mu$, then there exists $T>0$ such that the Hall-MHD system has a unique solution $(u, B)$ on $[0, T]$, with $(u, B, J)$ in $E_{2, r}(T)$.

Remark 3. Observe that for $h=0$, one recovers the statement of [16] pertaining to the classical incompressible MHD system.

The rest of the paper unfolds as follows. The next section is devoted to the proof of Theorem 2.1. In Section 4 we focus on the case $\mu=\nu$ and prove Theorem 2.2 by taking advantage of the cancellation property pointed out above. The proof of

\footnotetext{
${ }^{2}$ The reader may refer to Definition A.3 for the definition of 'tilde spaces'
} 
Theorem 2.3 is carried out in Section [5. For the reader's convenience, results concerning Besov spaces, Littlewood-Paley decomposition and commutator estimates are recalled in Appendix.

\section{Well-Posedness in general CRITICAl Besov SPACES With THIRD INDEX 1}

The present section is dedicated to proving Th. 2.1. Before starting, a fundamental observation (that will be also used in the next sections) is in order: the triplet $(u, B, P)$ satisfies the Hall-MHD system (1.1)-(1.3) with coefficients $(\mu, \nu, h)$ if and only if the rescaled triplet:

$$
(\widetilde{u}, \widetilde{B}, \widetilde{P})(t, x):=\frac{h}{\mu}\left(u, B, \frac{h}{\mu} P\right)\left(\frac{h^{2}}{\mu} t, h x\right)
$$

satisfies (1.1)-(1.3) with coefficients $(1,1, \nu / \mu)$.

Consequently, taking advantage of the scaling invariance of the homogeneous Besov norms (see Proposition A.2 (vi)), it is enough to prove the statement in the case where the viscosity $\mu$ and the Hall number $h$ are equal to 1 .

For expository purpose, we shall assume in addition that the magnetic resistivity $\nu$ is equal to 1 (to achieve the general case it is only a matter of changing the heat semi-group in the definition of $\mathcal{B}$ in (3.3) below accordingly).

Throughout this section and the following ones, we shall repeatedly use the fact that, as a consequence of Proposition A.2 (vii), one has the following equivalence of norms for all $s \in \mathbb{R}$ and $(p, r) \in[1,+\infty]^{2}$ :

$$
\|\nabla B\|_{\dot{B}_{p, r}^{s}} \sim\|J\|_{\dot{B}_{p, r}^{s}} \text { and }\|\nabla B\|_{\dot{H}^{s}}=\|J\|_{\dot{H}^{s}} .
$$

In order to establish the global existence of a solution of the Hall-MHD system in the case of small data, we shall first prove the corresponding result for the extended system (2.11). It relies on the following well known corollary of the fixed point theorem in complete metric spaces.

Lemma 3.1. Let $\left(X,\|\cdot\|_{X}\right)$ be a Banach space and $\mathcal{B}: X \times X \rightarrow X$, a bilinear continuous operator with norm $K$. Then, for all $y \in X$ such that $4 K\|y\|_{X}<1$, equation

$$
x=y+\mathcal{B}(x, x)
$$

has a unique solution $x$ in the ball $B\left(0, \frac{1}{2 K}\right)$. Besides, $x$ satisfies $\|x\|_{X} \leq 2\|y\|_{X}$.

We shall take for $X$ the set of triplets of (time dependent) divergence free vectorfields with components in $E_{p}$ endowed with the norm

$$
\|V\|_{X}:=\|V\|_{L^{1}\left(\dot{B}_{p, 1}^{\frac{3}{p}+1}\right)}+\|V\|_{L^{\infty}\left(\dot{B}_{p, 1}^{\frac{3}{p}-1}\right)} .
$$

Let $\left(e^{t \Delta}\right)_{t \geq 0}$ denote the heat semi-group defined in (A.3). We set $y: t \mapsto e^{t \Delta} U_{0}$ and define the bilinear functional $\mathcal{B}$ by the formula

$$
\mathcal{B}(V, W)(t)=\int_{0}^{t} e^{(t-\tau) \Delta} Q(V, W) d \tau .
$$

By virtue of (A.2), System (2.11) recasts in

$$
U(t)=y(t)+\mathcal{B}(U, U)(t) .
$$


In order to apply Lemma 3.1 it suffices to show that $y$ is small in $X$, and that $\mathcal{B}$ maps $X \times X$ to $X$. The former property holds true if Condition (2.1) is fulfilled for a small enough $c>0$, as Proposition A.4 ensures that $y$ belongs to $X$ and that

$$
\|y\|_{X} \leq C\left\|U_{0}\right\|_{\dot{B}_{p, 1}^{\frac{3}{p}-1}} \text {. }
$$

In order to prove the latter property, one can use the fact that, by virtue of Identity (2.5), Proposition A.2 (i), (iii), (vii), and Inequality (A.6), we have

$$
\begin{aligned}
\|\operatorname{div}(v \otimes w)\|_{\dot{B}_{p, 1}^{\frac{3}{p}-1}} & \lesssim\|v \otimes w\|_{\dot{B}_{p, 1}^{\frac{3}{p}}} \\
& \lesssim\|v\|_{\dot{B}_{p, 1}^{\frac{3}{p}}}\|w\|_{\dot{B}_{p, 1}^{\frac{3}{p}}}, \\
\left\|\operatorname{div}\left(\left(\operatorname{curl}^{-1} v\right) \otimes w\right)\right\|_{\dot{B}_{p, 1}^{\frac{3}{p}}} & =\left\|w \cdot \nabla\left(\operatorname{curl}^{-1} v\right)\right\|_{\dot{B}_{p, 1}^{\frac{3}{p}}} \\
& \lesssim\left\|\nabla \operatorname{curl}^{-1} v\right\|_{\dot{B}_{p, 1}^{\frac{3}{p}}}\|w\|_{\dot{B}_{p, 1}^{\frac{3}{p}}} \\
& \lesssim\|v\|_{\dot{B}_{p, 1}^{\frac{3}{p}}}\|w\|_{\dot{B}_{p, 1}^{\frac{3}{p}}} \\
& \lesssim\|v\|_{\dot{B}_{p, 1}^{\frac{3}{p}}}^{\frac{3}{p}-1}\|w\|_{\dot{B}_{p, 1}^{\frac{3}{p}-1}}^{\frac{3}{p}}\|v\|_{\dot{B}_{p, 1}^{\frac{3}{p}+1}}^{\frac{1}{2}}\|w\|_{\dot{B}_{p, 1}^{\frac{3}{p}+1}}^{\frac{1}{p}}
\end{aligned}
$$

and, since $\operatorname{div}\left(\operatorname{curl}^{-1} v\right)=0$, owing to Proposition A.2 (viii),

$$
\begin{aligned}
\left\|\operatorname{div}\left(w \otimes\left(\operatorname{curl}^{-1} v\right)\right)\right\|_{\dot{B}_{p, 1}^{\frac{3}{p}}} & =\left\|\left(\operatorname{curl}^{-1} v\right) \cdot \nabla w\right\|_{\dot{B}_{p, 1}^{\frac{3}{p}}} \\
& \lesssim\left\|\operatorname{curl}^{-1} v\right\|_{\dot{B}_{p, 1}^{\frac{3}{p}}}\|\nabla w\|_{\dot{B}_{p, 1}^{\frac{3}{p}}} \\
& \lesssim\|v\|_{\dot{B}_{p, 1}^{\frac{3}{p}-1}\|w\|_{\dot{B}_{p, 1}^{\frac{3}{p}+1}}}
\end{aligned}
$$

Hence, integrating on $\mathbb{R}_{+}$and observing that the Leray projector $\mathcal{P}$ maps $\dot{B}_{p, 1}^{\frac{3}{p}}$ to itself according to Proposition A.2 (vii), we get

$$
\begin{aligned}
&\left\|Q_{a}(v, w)\right\|_{L^{1}\left(\dot{B}_{p, 1}^{\frac{3}{p}}-1\right)} \lesssim\|\operatorname{div}(v \otimes w)+\operatorname{div}(w \otimes v)\|_{L^{1}\left(\dot{B}_{p, 1}^{\frac{3}{p}-1}\right)} \\
& \lesssim\|v\|_{X}\|w\|_{X} \\
&\left\|Q_{b}(v, w)\right\|_{L^{1}\left(\dot{B}_{p, 1}^{\frac{3}{p}}-1\right.}\left.=\|\operatorname{div}(v \otimes w)-\operatorname{div}(w \otimes v)\|_{L^{1}\left(\dot{B}_{p, 1}^{\frac{3}{p}}-1\right.}\right) \\
& \lesssim\|v\|_{X}\|w\|_{X} \\
&\left\|\nabla \times Q_{b}\left(\mathrm{Curl}^{-1} v, w\right)\right\|_{L^{1}\left(\dot{B}_{p, 1}^{\frac{3}{p}}-1\right)} \lesssim\left\|Q_{b}\left(\mathrm{Curl}^{-1} v, w\right)\right\|_{L^{1}\left(\dot{B}_{p, 1}^{\frac{3}{p}}\right)} \\
& \lesssim\left\|\operatorname{div}\left(\left(\mathrm{Curl}^{-1} v\right) \otimes w\right)\right\|_{L^{1}\left(\dot{B}_{p, 1}^{\frac{3}{p}}\right)}+\left\|\operatorname{div}\left(w \otimes\left(\operatorname{Curl}^{-1} v\right)\right)\right\|_{L^{1}\left(\dot{B}_{p, 1}^{\frac{3}{p}}\right)} \\
& \lesssim\|v\|_{X}\|w\|_{X} .
\end{aligned}
$$

Now, by definition of $\mathcal{B}(V, W)$, we have

$$
\left\{\begin{array}{l}
\partial_{t} \mathcal{B}(V, W)-\Delta \mathcal{B}(V, W)=Q(V, W) \\
\left.\mathcal{B}(V, W)\right|_{t=0}=0
\end{array}\right.
$$


Hence, by Proposition A.4 and the definition of $Q$ in (2.12), we get

$$
\begin{aligned}
& \|\mathcal{B}(V, W)\|_{X} \lesssim\left\|Q_{a}\left(V_{2}, W_{2}\right)-Q_{a}\left(V_{1}, W_{1}\right)\right\|_{L^{1}\left(\dot{B}_{p, 1}^{\frac{3}{p}-1}\right)} \\
& +\left\|Q_{b}\left(V_{2}, W_{3}-W_{1}\right)\right\|_{L^{1}\left(\dot{B}_{p, 1}^{\frac{3}{p}-1}\right)}+\left\|\nabla \times Q_{b}\left(\operatorname{curl}^{-1} V_{3}, W_{3}-W_{1}\right)\right\|_{L^{1}\left(\dot{B}_{p, 1}^{\frac{3}{p}-1}\right)} .
\end{aligned}
$$

Remembering (3.8)-(3.10), one can conclude that $\mathcal{B}$ maps $X \times X$ to $X$. Hence, System (2.11) has a global solution $(u, B, J)$ in $X$.

For completing the proof of the global existence for the original Hall-MHD system, we have to show that if $J_{0}=\nabla \times B_{0}$, then $J=\nabla \times B$ so that $(u, B)$ is indeed a distributional solution of (1.1)-(1.4). Now, we have

$$
\left(\partial_{t}-\Delta\right)(\nabla \times B-J)=\nabla \times Q_{b}\left(\operatorname{curl}^{-1}(\nabla \times B-J), J-u\right) .
$$

Remember that $J-u$ belongs to $L^{2}\left(\dot{B}_{p, 1}^{\frac{3}{p}}\right)$ (use interpolation for the space regularity and Hölder inequality for the time variable), that $J$ and $u$ are in $L_{T}^{2}\left(\dot{B}_{p, 1}^{\frac{3}{p}-1}\right)$ for all $T>0$ since they are in $L^{\infty}\left(\dot{B}_{p, 1}^{\frac{3}{p}-1}\right)$ and observe that $\nabla \times B$ is in $L^{2}\left(\dot{B}_{p, 1}^{\frac{3}{p}-1}\right)$. Therefore, from the definition of $Q_{b}$, the properties of continuity of operator curl ${ }^{-1}$, and product laws, we gather that $\nabla \times Q_{b}\left(\operatorname{curl}^{-1}(\nabla \times B-J), J-u\right)$ is in $L_{T}^{1}\left(\dot{B}_{p, 1}^{\frac{3}{p}-2}\right)$ for all $T>0$. Because $\left.(\nabla \times B-J)\right|_{t=0}=0$, Proposition A.4 thus guarantees that $\nabla \times B-J$ is in $\mathcal{C}\left([0, T] ; \dot{B}_{p, 1}^{\frac{3}{p}-2}\right)$ for all $T>0$. Furthermore, we have

$$
\begin{aligned}
&\|(\nabla \times B-J)(T)\|_{\dot{B}_{p, 1}^{\frac{3}{p}-2}}+\int_{0}^{T}\|\nabla \times B-J\|_{\dot{B}_{p, 1}^{\frac{3}{p}}} d t \\
& \leq C \int_{0}^{T}\|J-u\|_{\dot{B}_{p, 1}^{\frac{3}{p}}}\|\nabla \times B-J\|_{\dot{B}_{p, 1}^{\frac{3}{p}-1}} d t .
\end{aligned}
$$

The right-hand side may be handled by means of an interpolation inequality: we get for all $\varepsilon>0$,

$$
\|J-u\|_{\dot{B}_{p, 1}^{\frac{3}{p}}}\|\nabla \times B-J\|_{\dot{B}_{p, 1}^{\frac{3}{p}-1}} \leq \varepsilon\|\nabla \times B-J\|_{\dot{B}_{p, 1}^{\frac{3}{p}}}+C \varepsilon^{-1}\|J-u\|_{\dot{B}_{p, 1}^{\frac{3}{p}}}^{2}\|\nabla \times B-J\|_{\dot{B}_{p, 1}^{\frac{3}{p}-2}} .
$$

Hence, taking $\varepsilon$ small enough, then using Gronwall lemma ensures that $\|(\nabla \times B-$ $J)(t) \|_{\dot{B}_{p, 1}^{\frac{3}{p}-2}}=0$, whence $\nabla \times B-J \equiv 0$ a.e. on $\mathbb{R}_{+} \times \mathbb{R}^{3}$. This yields the existence part of Theorem 2.1 in the small data case.

Let us explain how the above arguments have to be modified so as to prove local existence in the case where only $J_{0}$ is small. The idea is to control the existence time according to the solution $U^{L}$ of the heat equation:

$$
\left\{\begin{array}{l}
\partial_{t} U^{L}-\Delta U^{L}=0, \\
\left.U^{L}\right|_{t=0}=U_{0}
\end{array}\right.
$$

By Proposition A.4 we have

$$
\left\|J^{L}\right\|_{L_{T}^{\infty}\left(\dot{B}_{p, 1}^{\frac{3}{p}-1}\right)} \leq C\left\|J_{0}\right\|_{\dot{B}_{p, 1}^{\frac{3}{p}-1}}
$$

and, using also the dominated convergence theorem yields

$$
\lim _{T \rightarrow 0}\left\|U^{L}\right\|_{L_{T}^{\rho}\left(\dot{B}_{p, 1}^{\frac{3}{p}+\frac{2}{\rho}-1}\right)}=0, \text { whenever } 1 \leq \rho<\infty .
$$


Clearly, $U$ is a solution of (2.11) on $[0, T] \times \mathbb{R}^{3}$ with data $U_{0}$ if and only if

$$
U:=U^{L}+\widetilde{U}
$$

with, for all $t \in[0, T]$,

$$
\widetilde{U}(t):=\int_{0}^{t} e^{(t-\tau) \Delta}\left(Q\left(\widetilde{U}, U^{L}\right)+Q\left(U^{L}, \widetilde{U}\right)+Q(\widetilde{U}, \widetilde{U})+Q\left(U^{L}, U^{L}\right)\right) d \tau .
$$

Then, proving local existence relies on the following generalization of Lemma 3.1

Lemma 3.2. Let $\left(X,\|\cdot\|_{X}\right)$ be a Banach space, $\mathcal{B}: X \times X \rightarrow X$, a bilinear continuous operator with norm $K$ and $\mathcal{L}: X \rightarrow X$, a continuous linear operator with norm $M<1$. Let $y \in X$ satisfy $4 K\|y\|_{X}<(1-M)^{2}$. Then, equation

$$
x=y+\mathcal{L}(x)+\mathcal{B}(x, x)
$$

has a unique solution $x$ in the ball $B\left(0, \frac{1-M}{2 K}\right)$.

Take $\mathcal{B}$ as in (3.3), set $y:=\mathcal{B}\left(U^{L}, U^{L}\right)$ and define the linear map $\mathcal{L}$ by

$$
\mathcal{L}(V):=\mathcal{B}\left(V, U^{L}\right)+\mathcal{B}\left(U^{L}, V\right) .
$$

Our problem recasts in

$$
\widetilde{U}=y+\mathcal{L}(\widetilde{U})+\mathcal{B}(\widetilde{U}, \widetilde{U}) .
$$

For $X$, we now take the space (denoted by $X_{T}$ ) of triplets of divergence free vectorfields with components in $E_{p}(T)$. Then, arguing as for getting (3.5), (3.6), integrating on $[0, T]$ and using Cauchy-Schwarz inequality, we get

$$
\|\operatorname{div}(v \otimes w)\|_{L_{T}^{1}\left(\dot{B}_{p, 1}^{\frac{3}{p}}-1\right)}+\left\|\operatorname{div}\left(\operatorname{curl}^{-1} v \otimes w\right)\right\|_{L_{T}^{1}\left(\dot{B}_{p, 1}^{\frac{3}{p}}\right)} \lesssim\|v\|_{L_{T}^{2}\left(\dot{B}_{p, 1}^{\frac{3}{p}}\right)}\|w\|_{L_{T}^{2}\left(\dot{B}_{p, 1}^{\frac{3}{p}}\right)} .
$$

Hence, using also (3.7) and the definition of $\mathcal{B}(V, W)$, we end up with

$$
\begin{aligned}
& \|\mathcal{B}(V, W)\|_{X_{T}} \lesssim\|V\|_{L_{T}^{2}\left(\dot{B}_{p, 1}^{\frac{3}{p}}\right)}\|W\|_{L_{T}^{2}\left(\dot{B}_{p, 1}^{\frac{3}{p}}\right)} \\
& +\left(\left\|W_{1}\right\|_{L_{T}^{1}\left(\dot{B}_{p, 1}^{\frac{3}{p}+1}\right)}+\left\|W_{3}\right\|_{L_{T}^{1}\left(\dot{B}_{p, 1}^{\frac{3}{p}+1}\right)}\right)\left\|V_{3}\right\|_{L_{T}^{\infty}\left(\dot{B}_{p, 1}^{\frac{3}{p}-1}\right)} .
\end{aligned}
$$

For justifying that $\mathcal{L}$ defined in (3.13) is indeed a continuous linear operator on $X_{T}$ with small norm if $T \rightarrow 0$, the troublemakers in the right-hand side of (3.15) are

$$
\|\widetilde{u}\|_{L_{T}^{1}\left(\dot{B}_{p, 1}^{\frac{3}{p}+1}\right)}\left\|J^{L}\right\|_{L_{T}^{\infty}\left(\dot{B}_{p, 1}^{\frac{3}{p}-1}\right)} \text { and }\|\widetilde{J}\|_{L_{T}^{1}\left(\dot{B}_{p, 1}^{\frac{3}{p}+1}\right)}\left\|J^{L}\right\|_{L_{T}^{\infty}\left(\dot{B}_{p, 1}^{\frac{3}{p}}-1\right.}
$$

since, for large $J_{0}$, the term $\left\|J^{L}\right\|_{L_{T}^{\infty}\left(\dot{B}_{p, 1}^{\frac{3}{p}-1}\right)}$ need not to be small. One thus have to assume that $\left\|J_{0}\right\|_{\dot{B}_{p, 1}^{\frac{3}{p}-1}}$ is small so as to guarantee that the norm of $\mathcal{L}$ is smaller than 1 for $T$ small enough. Then, one can conclude thanks to Lemma 3.2, to the local-in-time existence statement of Theorem 2.1 .

To prove the uniqueness part of Theorem 2.1. Consider two solutions $\left(u^{1}, B^{1}\right)$ and $\left(u^{2}, B^{2}\right)$ of (1.1)-1.3) emanating from the same data, and denote by $U^{1}$ and $U^{2}$ the corresponding solutions of the extended system (2.11). Since one can take (with no loss of generality) for $U^{2}$ the solution built previously, and as $\left\|J_{0}\right\|_{\dot{B}_{p, 1}^{\frac{3}{p}-1}} \leq c$ is assumed, we have

$$
\left\|J^{2}\right\|_{X_{T}} \leq 2 c
$$


Denoting $\delta U:=U^{2}-U^{1}$, we find that $\delta U$ satisfies

$$
\partial_{t} ठ U-\Delta \delta=Q\left(U^{2}, \delta U\right)+Q\left(\delta U, U^{1}\right)
$$

with $\left.\delta U\right|_{t=0}=0$, and thus

$$
\delta U=\mathcal{B}\left(U^{2}, \delta U\right)+\mathcal{B}\left(\delta U, U^{1}\right) .
$$

Arguing as in the proof of (3.15) yields

$$
\begin{aligned}
& \left\|\mathcal{B}\left(U^{2}, \delta U\right)\right\|_{X_{T}} \lesssim \int_{0}^{T}\left\|U^{2}\right\|_{\dot{B}_{p, 1}^{\frac{3}{p}}}\|\delta U\|_{\dot{B}_{p, 1}^{\frac{3}{p}}} d t+\int_{0}^{T}\left\|J^{2}\right\|_{\dot{B}_{p, 1}^{\frac{3}{p}-1}}\|\delta U\|_{\dot{B}_{p, 1}^{\frac{3}{p}+1}} d t \\
& \lesssim \int_{0}^{T}\left\|U^{2}\right\|_{\dot{B}_{p, 1}^{\frac{3}{p}}}\|\delta U\|_{\dot{B}_{p, 1}^{\frac{3}{p}-1}}^{\frac{1}{2}}\|\delta U\|_{\dot{B}_{p, 1}^{\frac{3}{p}+1}}^{\frac{1}{p}} d t+\int_{0}^{T}\left\|J^{2}\right\|_{\dot{B}_{p, 1}^{\frac{3}{p}-1}}\|\delta U\|_{\dot{B}_{p, 1}^{\frac{3}{p}+1}} d t
\end{aligned}
$$

whence there exists $C>0$ such that for all $\eta>0$,

$$
\begin{aligned}
\left\|\mathcal{B}\left(U^{2}, \delta U\right)\right\|_{X_{T}} \leq\left(\eta+C\left\|J^{2}\right\|_{L_{T}^{\infty}\left(\dot{B}_{p, 1}^{\frac{3}{p}-1}\right)}\right)\|\delta U\|_{L_{T}^{1}\left(\dot{B}_{p, 1}^{\frac{3}{p}+1}\right)} & \\
& +C \eta^{-1} \int_{0}^{T}\left\|U^{2}\right\|_{\dot{B}_{p, 1}^{\frac{3}{p}}}^{2}\|\delta U\|_{\dot{B}_{p, 1}^{\frac{3}{p}-1}} d t .
\end{aligned}
$$

Similarly, we have

$$
\begin{aligned}
\left\|\mathcal{B}\left(\delta U, U^{1}\right)\right\|_{X_{T}} & \leq C\left(\int_{0}^{T}\left\|U^{1}\right\|_{\dot{B}_{p, 1}^{\frac{3}{p}}}\|\delta U\|_{\dot{B}_{p, 1}^{\frac{3}{p}}} d t+\int_{0}^{T}\left\|U^{1}\right\|_{\dot{B}_{p, 1}^{\frac{3}{p}+1}}\|\delta J\|_{\dot{B}_{p, 1}^{\frac{3}{p}-1}} d t\right) \\
& \leq \eta\|\delta U\|_{L_{T}^{1}\left(\dot{B}_{p, 1}^{\frac{3}{p}+1}\right)}+C \int_{0}^{T}\left(\left\|U^{1}\right\|_{\dot{B}_{p, 1}^{\frac{3}{p}+1}}+\eta^{-1}\left\|U^{1}\right\|_{\dot{B}_{p, 1}^{\frac{3}{p}}}^{2}\|\delta U\|_{\dot{B}_{p, 1}^{\frac{3}{p}-1}} d t .\right.
\end{aligned}
$$

Hence, taking $\eta$ small enough, and remembering (3.16), one gets

$$
\|\delta U\|_{X_{T}} \leq C \int_{0}^{T}\left(\left\|U^{1}\right\|_{\dot{B}_{p, 1}^{\frac{3}{p}+1}}+\left\|U^{1}\right\|_{\dot{B}_{p, 1}^{\frac{3}{p}}}^{2}+\left\|U^{2}\right\|_{\dot{B}_{p, 1}^{\frac{3}{p}}}^{2}\right)\|\delta U\|_{\dot{B}_{p, 1}^{\frac{3}{p}-1}} d t .
$$

Gronwall lemma thus implies that $\delta U \equiv 0$ in $X_{T}$, whence uniqueness on $[0, T] \times \mathbb{R}^{3}$. Of course, in the case where the data are small, then $J^{2}$ remains small for all $T>0$, and one gets uniqueness on $\mathbb{R}_{+} \times \mathbb{R}^{3}$.

Let us finally justify the propagation of Sobolev regularity in the case where, additionally, $\left(u_{0}, B_{0}\right)$ is in $H^{s} \times H^{r}$ with $(r, s)$ satisfying (2.3). For expository purpose, assume that the data fulfill (2.1) (the case where only $J_{0}$ is small being left to the reader). Our aim is to prove that the solution $(u, B)$ we constructed above satisfies

$$
(u, B) \in \mathcal{C}_{b}\left(\mathbb{R}_{+} ; H^{s} \times H^{r}\right) \quad \text { and } \quad(\nabla u, \nabla B) \in L^{2}\left(\mathbb{R}_{+} ; H^{s} \times H^{r}\right) .
$$

For the time being, let us assume that $(u, B)$ is smooth. Then, taking the $L^{2}$ scalar product of (1.1) and (1.3) by $u$ and $B$, respectively, adding up the resulting identities, and using the fact that

$$
(\nabla \times(J \times B), B)=(J \times B, J)=0,
$$

one gets the following energy balance:

$$
\frac{1}{2} \frac{d}{d t}\left(\|u\|_{L^{2}}^{2}+\|B\|_{L^{2}}^{2}\right)+\|\nabla u\|_{L^{2}}^{2}+\|\nabla B\|_{L^{2}}^{2}=0 .
$$


Since $\|z\|_{\dot{H}^{a}}=\left\|\Lambda^{a} z\right\|_{L^{2}}$ and $\|z\|_{H^{a}} \sim\|z\|_{L^{2}}+\|z\|_{\dot{H}^{a}}$, in order to prove estimates in $H^{s} \times H^{r}$, it suffices to get a suitable control on $\left\|\Lambda^{s} u\right\|_{L^{2}}$ and on $\left\|\Lambda^{r} B\right\|_{L^{2}}$. To this end, apply $\Lambda^{s}$ to (1.1), then take the $L^{2}$ scalar product with $\Lambda^{s} u$ :

$$
\begin{aligned}
\frac{1}{2} \frac{d}{d t}\left\|\Lambda^{s} u\right\|_{L^{2}}^{2}+\left\|\Lambda^{s} \nabla u\right\|_{L^{2}}^{2} & =\left(\Lambda^{s}(B \cdot \nabla B), \Lambda^{s} u\right)-\left(\Lambda^{s}(u \cdot \nabla u), \Lambda^{s} u\right) \\
& =: A_{1}+A_{2} .
\end{aligned}
$$

Similarly, apply $\Lambda^{r}$ to (1.3) and take the $L^{2}$ scalar product with $\Lambda^{r} B$ to get:

$$
\begin{aligned}
\frac{1}{2} \frac{d}{d t}\left\|\Lambda^{r} B\right\|_{L^{2}}^{2}+\left\|\Lambda^{r} \nabla B\right\|_{L^{2}}^{2} & =\left(\Lambda^{r}(u \times B), \Lambda^{r} J\right)-\left(\Lambda^{r}(J \times B), \Lambda^{r} J\right) \\
& =: A_{3}+A_{4} .
\end{aligned}
$$

To bound $A_{1}, A_{2}, A_{3}$ and $A_{4}$, we shall use repeatedly the following classical tame estimate in homogeneous Sobolev spaces:

$$
\left\|\Lambda^{\sigma}(f g)\right\|_{L^{2}} \lesssim\|f\|_{L^{\infty}}\left\|\Lambda^{\sigma} g\right\|_{L^{2}}+\|g\|_{L^{\infty}}\left\|\Lambda^{\sigma} f\right\|_{L^{2}}, \quad \sigma \geq 0 .
$$

Using first the Cauchy-Schwarz inequality, then (3.18), the fact that $s \leq r \leq 1+s$ and Young inequality, we readily get

$$
\begin{aligned}
\left|A_{1}\right| & \leq C\left(\left\|\Lambda^{s} B\right\|_{L^{2}}\|\nabla B\|_{L^{\infty}}+\|B\|_{L^{\infty}}\left\|\Lambda^{s} \nabla B\right\|_{L^{2}}\right)\|u\|_{H^{s}} \\
& \leq C\left(\|B\|_{H^{s}}^{2}+\|u\|_{H^{s}}^{2}\right)\|\nabla B\|_{L^{\infty}}+\frac{1}{8}\|\nabla B\|_{H^{r}}^{2}+C\|B\|_{L^{\infty}}^{2}\|u\|_{H^{s}}^{2}, \\
\left|A_{2}\right| & \leq C\|\nabla u\|_{L^{\infty}}\|u\|_{H^{s}}^{2}, \\
\left|A_{3}\right| & \leq C\left\|\Lambda^{r}(u \times B)\right\|_{L^{2}}\left\|\Lambda^{r} J\right\|_{L^{2}} \\
& \leq C\left(\left\|\Lambda^{r} u\right\|_{L^{2}}^{2}\|B\|_{L^{\infty}}^{2}+\left\|\Lambda^{r} B\right\|_{L^{2}}^{2}\|u\|_{L^{\infty}}^{2}\right)+\frac{1}{8}\|\nabla B\|_{H^{r}}^{2} \\
& \leq C\left(\|u\|_{L^{2}}^{2}+\|\nabla u\|_{H^{s}}^{2}\right)\|B\|_{L^{\infty}}^{2}+C\|B\|_{H^{r}}^{2}\|u\|_{L^{\infty}}^{2}+\frac{1}{8}\|\nabla B\|_{H^{r}}^{2} \\
\left|A_{4}\right| & \leq C\|J \times B\|_{H^{r}}\|J\|_{H^{r}} \\
& \leq C\left(\|J\|_{H^{r}}^{2}\|B\|_{L^{\infty}}+\|J\|_{L^{\infty}}\|B\|_{H^{r}}\|J\|_{H^{r}}\right) \\
& \leq C\|B\|_{L^{\infty}}\|\nabla B\|_{H^{r}}^{2}+C\|J\|_{L^{\infty}}^{2}\|B\|_{H^{r}}^{2}+\frac{1}{8}\|J\|_{H^{r}}^{2} .
\end{aligned}
$$

Putting the above estimates and (3.17) together, and using the fact that $\|B\|_{L^{\infty}}$ is small since, according to Proposition A.2 and the first part of the proof, we have

$$
\|B\|_{L^{\infty}} \lesssim\|B\|_{\dot{B}_{p, 1}^{\frac{3}{p}}} \lesssim\|J\|_{\dot{B}_{p, 1}^{\frac{3}{p}-1}} \lesssim\left\|\left(u_{0}, B_{0}, J_{0}\right)\right\|_{\dot{B}_{p, 1}^{\frac{3}{p}-1}},
$$

one gets

$$
\frac{1}{2} \frac{d}{d t}\left(\|u\|_{H^{s}}^{2}+\|B\|_{H^{r}}^{2}\right)+\|\nabla u\|_{H^{s}}^{2}+\|\nabla B\|_{H^{r}}^{2} \leq C\left(\|u\|_{H^{s}}^{2}+\|B\|_{H^{r}}^{2}\right) S(t),
$$

with

$$
S(t):=\|\nabla u(t)\|_{L^{\infty}}+\|\nabla B(t)\|_{L^{\infty}}+\|u(t)\|_{L^{\infty}}^{2}+\|B(t)\|_{L^{\infty}}^{2}+\|J(t)\|_{L^{\infty}}^{2} .
$$

By Gronwall's inequality, we conclude that for all $t \geq 0$,

$$
\begin{aligned}
\|u(t)\|_{H^{s}}^{2}+\|B(t)\|_{H^{r}}^{2}+\int_{0}^{t}\left(\|\nabla u(\tau)\|_{H^{s}}^{2}+\|\nabla B(\tau)\|_{H^{r}}^{2}\right) d \tau \\
\quad \leq\left(\left\|u_{0}\right\|_{H^{s}}^{2}+\left\|B_{0}\right\|_{H^{r}}^{2}\right) \exp \left(C \int_{0}^{t} S(\tau) d \tau\right) .
\end{aligned}
$$


As $\int_{0}^{t} S(\tau) d \tau$ is bounded thanks to the first part of the theorem and embedding (use Proposition A.2 (ii)), we get a control of the Sobolev norms for all time.

Let us briefly explain how those latter computations may be made rigorous. Let us consider data $\left(u_{0}, B_{0}\right)$ fulfilling (2.1) and such that, additionally, we have $u_{0}$ in $H^{s}$ and $B_{0}$ in $H^{r}$ with $(r, s)$ satisfying (2.3). Then, there exists a sequence $\left(u_{0}^{n}, B_{0}^{n}\right)$ in the Schwartz space $\mathcal{S}$ such that

$$
\left(u_{0}^{n}, B_{0}^{n}\right) \rightarrow\left(u_{0}, B_{0}\right) \text { in }\left(\dot{B}_{p, 1}^{\frac{3}{p}-1} \cap H^{s}\right) \times\left(\dot{B}_{p, 1}^{\frac{3}{p}-1} \cap B_{p, 1}^{\frac{3}{p}} \cap H^{r}\right) .
$$

The classical well-posedness theory in Sobolev spaces (see e.g. 8]) ensures that the Hall-MHD system with data $\left(u_{0}^{n}, B_{0}^{n}\right)$ has a unique maximal solution $\left(u^{n}, B^{n}\right)$ on some interval $\left[0, T^{n}\right)$ belonging to all Sobolev spaces. For that solution, the previous computations hold, and one ends up for all $t<T^{n}$ with

$$
\begin{aligned}
\left\|u^{n}(t)\right\|_{H^{s}}^{2}+\left\|B^{n}(t)\right\|_{H^{r}}^{2}+\int_{0}^{t}\left(\left\|\nabla u^{n}(\tau)\right\|_{H^{s}}^{2}+\left\|\nabla B^{n}(\tau)\right\|_{H^{r}}^{2}\right) d \tau & \\
& \leq\left(\left\|u_{0}^{n}\right\|_{H^{s}}^{2}+\left\|B_{0}^{n}\right\|_{H^{r}}^{2}\right) \exp \left(C \int_{0}^{t} S^{n}(\tau) d \tau\right),
\end{aligned}
$$

where

$$
S^{n}(t):=\left\|\nabla u^{n}(t)\right\|_{L^{\infty}}+\left\|\nabla B^{n}(t)\right\|_{L^{\infty}}+\left\|u^{n}(t)\right\|_{L^{\infty}}^{2}+\left\|B^{n}(t)\right\|_{L^{\infty}}^{2}+\left\|J^{n}(t)\right\|_{L^{\infty}}^{2} .
$$

Since the regularized data $\left(u_{0}^{n}, B_{0}^{n}\right)$ fulfill (2.1) for large enough $n$, they generate a global solution $\left(\widetilde{u}^{n}, \widetilde{B}^{n}\right)$ in $E_{p}$ which, actually, coincides with $\left(u^{n}, B^{n}\right)$ on $\left[0, T^{n}\right)$ by virtue of the uniqueness result that has been proved before. Therefore, $S^{n}$ belongs to $L^{1}\left(0, T^{n}\right)$ and thus $\left(u^{n}, B^{n}\right)$ is in $L^{\infty}\left(0, T^{n} ; H^{s} \times H^{r}\right)$. Combining with the continuation argument of e.g. [8, one can conclude that $T^{n}=+\infty$.

At this stage, one can assert that:

i) $\left(u^{n}, B^{n}, J^{n}\right)_{n \in \mathbb{N}}$ is bounded in $E_{p}$;

ii) $\left(u^{n}, B^{n}\right)_{n \in \mathbb{N}}$ is bounded in $\mathcal{C}\left(\mathbb{R}_{+} ; H^{s} \times H^{r}\right)$ and $\left(\nabla u^{n}, \nabla B^{n}\right)_{n \in \mathbb{N}}$ is bounded in $L^{2}\left(\mathbb{R}_{+} ; H^{s} \times H^{r}\right)$.

Hence, up to subsequence,

i) $\left(u^{n}, B^{n}, J^{n}\right)$ converges weakly $*$ in $E_{p}$;

ii) $\left(u^{n}, B^{n}\right)$ converges weakly $*$ in $L^{\infty}\left(\mathbb{R}_{+} ; H^{s} \times H^{r}\right)$;

iii) $\left(\nabla u^{n}, \nabla B^{n}\right)$ converges weakly in $L^{2}\left(\mathbb{R}_{+} ; H^{s} \times H^{r}\right)$.

Clearly, a small variation of the proof of uniqueness in $E_{p}$ allows to prove the continuity of the flow map. Hence, given that $\left(u_{0}^{n}, B_{0}^{n}, J_{0}^{n}\right)$ converges to $\left(u_{0}, B_{0}, J_{0}\right)$ in $\dot{B}_{p, 1}^{\frac{3}{p}-1}$, one gets $\left(u^{n}, B^{n}, J^{n}\right) \rightarrow(u, B, J)$ strongly in $E_{p}$, where $(u, B, J)$ stands for the solution of (2.11) with data $\left(u_{0}, B_{0}, J_{0}\right)$.

Since the weak convergence results listed above imply the convergence in the sense of distributions, one can conclude that the weak limit coincides with the strong one in $E_{p}$. Hence $(u, B)$ (resp. $\left.(\nabla u, \nabla B)\right)$ is indeed in $L^{\infty}\left(\mathbb{R}_{+} ; H^{s} \times H^{r}\right)$ (resp. $\left.L^{2}\left(\mathbb{R}_{+} ; H^{s} \times H^{r}\right)\right)$. Then, looking at $(u, B)$ as the solution of a heat equation with right-hand side in $L^{2}\left(\mathbb{R}_{+} ; H^{s-1} \times H^{r-1}\right)$ yields the time continuity with values in Sobolev spaces (use for instance Proposition A.4). This completes the proof of Theorem 2.1. 
4. LoCAL EXISTENCE FOR LARGE DATA IN $\dot{B}_{2,1}^{\frac{1}{2}}$, AND BLOW-UP CRITERIA

Proving Theorem 2.2 is based on a priori estimates in the space $E_{2}(T)$ for smooth solutions $(u, B, v)$ of (2.14). Those estimates will be obtained by implementing an energy method on (2.14) after localization in the Fourier space. A slight modification of the method will yield uniqueness and blow-up criteria.

Throughout this section and the following one, we shall take advantage of the rescaling (3.1), so as to reduce our study to the case $\mu=\nu=h=1$ (remember that we have $\mu / \nu=1$ in Theorem 2.2).

\section{First step: A priori estimates.}

Our main aim here is to prove the following result.

Proposition 4.1. Consider a smooth solution $(u, B, P)$ to the Hall-MHD System on $[0, T] \times \mathbb{R}^{3}$ for some $T>0$, and denote $v:=u-\nabla \times B$. Let $u^{L}:=e^{t \Delta} u_{0}$, $B^{L}:=e^{t \Delta} B_{0}, v^{L}:=e^{t \Delta} v_{0}$ and $(\widetilde{u}, \widetilde{B}, \widetilde{v}):=\left(u-u^{L}, B-B^{L}, v-v^{L}\right)$. Let

$$
\begin{aligned}
& c_{1}(t):=\left\|v^{L}(t)\right\|_{\dot{B}_{2,1}^{\frac{5}{2}},} \\
& c_{2}(t):=\left\|u^{L}(t)\right\|_{\dot{B}_{2,1}^{\frac{3}{2}}}^{2}+\left\|B^{L}(t)\right\|_{\dot{B}_{2,1}^{\frac{3}{2}}}^{2}+\left(\left\|u_{0}\right\|_{\dot{B}_{2,1}^{\frac{1}{2}}}+\left\|v_{0}\right\|_{\dot{B}_{2,1}^{\frac{1}{2}}}\right)\left\|v^{L}(t)\right\|_{\dot{B}_{2,1}^{\frac{5}{2}}} .
\end{aligned}
$$

There exist three positive constants $\kappa, C$ and $C_{1}$ such that if

$$
\int_{0}^{T} c_{2}(\tau) e^{C \int_{\tau}^{T} c_{1}\left(\tau^{\prime}\right) d \tau^{\prime}} d \tau<\kappa
$$

then we have

$$
\begin{aligned}
& \|(\widetilde{u}, \widetilde{B}, \widetilde{v})\|_{L_{T}^{\infty}\left(\dot{B}_{2,1}^{\frac{1}{2}}\right)}+C_{1}\|(\widetilde{u}, \widetilde{B}, \widetilde{v})\|_{L_{T}^{1}\left(\dot{B}_{2,1}^{\frac{5}{2}}\right)} \leq C \kappa \text { and } \\
& \|(u, B, v)\|_{L_{T}^{\infty}\left(\dot{B}_{2,1}^{\frac{1}{2}}\right)}+C_{1}\|(u, B, v)\|_{L_{T}^{1}\left(\dot{B}_{2,1}^{\frac{5}{2}}\right)} \leq\left\|\left(u_{0}, B_{0}, v_{0}\right)\right\|_{\dot{B}_{2,1}^{\frac{1}{2}}}+C \kappa .
\end{aligned}
$$

Proof. From (A.3), Plancherel identity and the definition of $\|\cdot\|_{\dot{B}_{2,1}^{s}}$, we have for some universal constant $C_{1}$,

$$
\|z\|_{L_{T}^{\infty}\left(\dot{B}_{2,1}^{\frac{1}{2}}\right)}+C_{1}\|z\|_{L_{T}^{1}\left(\dot{B}_{2,1}^{\frac{5}{2}}\right)} \leq\left\|z_{0}\right\|_{\dot{B}_{2,1}^{\frac{1}{2}}} \text { for } z=u^{L}, B^{L}, v^{L} .
$$

Hence Inequality (4.3) follows from Inequality (4.2).

In order to prove (4.2), we use the fact that $(\widetilde{u}, \widetilde{B}, \widetilde{v}, Q)$ satisfies

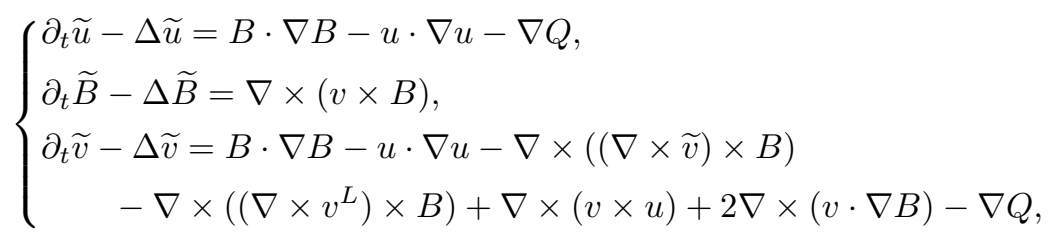

with null initial condition.

Apply operator $\dot{\Delta}_{j}$ to both sides of (4.5), then take the $L^{2}$ scalar product with $\dot{\Delta}_{j} \widetilde{u}, \dot{\Delta}_{j} \widetilde{B}, \dot{\Delta}_{j} \widetilde{v}$, respectively. To handle the third equation of (4.5), use that

$$
\nabla \times \dot{\Delta}_{j}((\nabla \times \widetilde{v}) \times B)=\nabla \times\left(\left[\dot{\Delta}_{j}, B \times\right](\nabla \times \widetilde{v})\right)+\nabla \times\left(B \times \dot{\Delta}_{j}(\nabla \times \widetilde{v})\right),
$$


and that the $L^{2}$ scalar product of the last term with $\dot{\Delta}_{j} \widetilde{v}$ is 0 . Then, we get

$$
\begin{aligned}
& \frac{1}{2} \frac{d}{d t}\left\|\dot{\Delta}_{j} \widetilde{u}\right\|_{L^{2}}^{2}+\left\|\nabla \dot{\Delta}_{j} \widetilde{u}\right\|_{L^{2}}^{2} \leq\left(\left\|\dot{\Delta}_{j}(B \cdot \nabla B)\right\|_{L^{2}}+\left\|\dot{\Delta}_{j}(u \cdot \nabla u)\right\|_{L^{2}}\right)\left\|\dot{\Delta}_{j} \widetilde{u}\right\|_{L^{2}}, \\
& \frac{1}{2} \frac{d}{d t}\left\|\dot{\Delta}_{j} \widetilde{B}\right\|_{L^{2}}^{2}+\left\|\nabla \dot{\Delta}_{j} \widetilde{B}\right\|_{L^{2}}^{2} \leq\left\|\nabla \times \dot{\Delta}_{j}(v \times B)\right\|_{L^{2}}\left\|\dot{\Delta}_{j} \widetilde{B}\right\|_{L^{2}}, \\
& \frac{1}{2} \frac{d}{d t}\left\|\dot{\Delta}_{j} \widetilde{v}\right\|_{L^{2}}^{2}+\left\|\nabla \dot{\Delta}_{j} \widetilde{v}\right\|_{L^{2}}^{2} \leq\left(\left\|\dot{\Delta}_{j}(B \cdot \nabla B)\right\|_{L^{2}}+\left\|\dot{\Delta}_{j}(u \cdot \nabla u)\right\|_{L^{2}}\right)\left\|\dot{\Delta}_{j} \widetilde{v}\right\|_{L^{2}} \\
&+\left(\left\|\left[\dot{\Delta}_{j}, B \times\right](\nabla \times \widetilde{v})\right\|_{L^{2}}+\left\|\dot{\Delta}_{j}\left(\left(\nabla \times v^{L}\right) \times B\right)\right\|_{L^{2}}+\left\|\dot{\Delta}_{j}(v \times u)\right\|_{L^{2}}\right. \\
&\left.+2\left\|\dot{\Delta}_{j}(v \cdot \nabla B)\right\|_{L^{2}}\right)\left\|\nabla \times \dot{\Delta}_{j} \widetilde{v}\right\|_{L^{2}} .
\end{aligned}
$$

Hence, using Bernstein inequalities, one can deduce after time integration that for some universal constants $C_{1}$ and $C_{2}$,

$$
\begin{aligned}
\left\|\left(\dot{\Delta}_{j} \widetilde{u}, \dot{\Delta}_{j} \widetilde{B}, \dot{\Delta}_{j} \widetilde{v}\right)(t)\right\|_{L^{2}} & +C_{1} 2^{2 j} \int_{0}^{t}\left\|\left(\dot{\Delta}_{j} \widetilde{u}, \dot{\Delta}_{j} \widetilde{B}, \dot{\Delta}_{j} \widetilde{v}\right)\right\|_{L^{2}} d \tau \\
\leq \int_{0}^{t}\left(\left\|\dot{\Delta}_{j}(B \cdot \nabla B)\right\|_{L^{2}}\right. & +\left\|\dot{\Delta}_{j}(u \cdot \nabla u)\right\|_{L^{2}}+C_{2} 2^{j}\left(\left\|\left[\dot{\Delta}_{j}, B \times\right](\nabla \times \widetilde{v})\right\|_{L^{2}}\right. \\
+\left\|\dot{\Delta}_{j}\left(\left(\nabla \times v^{L}\right) \times B\right)\right\|_{L^{2}}+\left\|\dot{\Delta}_{j}(v \times u)\right\|_{L^{2}} & \\
& \left.\left.\left.\quad\left\|\dot{\Delta}_{j}(v \cdot \nabla B)\right\|_{L^{2}}+\left\|\dot{\Delta}_{j}(v \times B)\right\|_{L^{2}}\right) d \tau\right)\right) d \tau
\end{aligned}
$$

Multiplying both sides of (4.6) by $2^{\frac{j}{2}}$ and summing up over $j \in \mathbb{Z}$, we obtain that

$$
\begin{aligned}
& \|(\widetilde{u}, \widetilde{B}, \widetilde{v})(t)\|_{\dot{B}_{2,1}^{\frac{1}{2}}}+C_{1} \int_{0}^{t}\|(\widetilde{u}, \widetilde{B}, \widetilde{v})\|_{\dot{B}_{2,1}^{\frac{5}{2}}} d \tau \\
& \quad \leq C_{2} \int_{0}^{t}\left(\|B \cdot \nabla B\|_{\dot{B}_{2,1}^{\frac{1}{2}}}+\|u \cdot \nabla u\|_{\dot{B}_{2,1}^{\frac{1}{2}}}+\|v \times B\|_{\dot{B}_{2,1}^{\frac{3}{2}}}+\|v \times u\|_{\dot{B}_{2,1}^{\frac{3}{2}}}\right. \\
& \left.+\|v \cdot \nabla B\|_{\dot{B}_{2,1}^{\frac{3}{2}}}+\left\|\left(\nabla \times v^{L}\right) \times B\right\|_{\dot{B}_{2,1}^{\frac{3}{2}}}+\sum_{j} 2^{\frac{3 j}{2}}\left\|\left[\dot{\Delta}_{j}, B \times\right](\nabla \times \widetilde{v})\right\|_{L^{2}}\right) d \tau .
\end{aligned}
$$

Using (A.6), Proposition A.2 (i), (ii), (iii) and Young's inequality yields

$$
\begin{aligned}
& \|B \cdot \nabla B\|_{\dot{B}_{2,1}^{\frac{1}{2}}} \lesssim\left\|B^{L}\right\|_{\dot{B}_{2,1}^{\frac{3}{2}}}^{2}+\|\tilde{B}\|_{\dot{B}_{2,1}^{\frac{3}{2}}}^{2} \\
& \lesssim\left\|B^{L}\right\|_{\dot{B}_{2,1}^{\frac{3}{2}}}^{2}+\|\widetilde{B}\|_{\dot{B}_{2,1}^{\frac{1}{2}}}\|\widetilde{B}\|_{\dot{B}_{2,1}^{\frac{5}{2}}}, \\
& \|u \cdot \nabla u\|_{\dot{B}_{2,1}^{\frac{1}{2}}}+\|v \times B\|_{\dot{B}_{2,1}^{\frac{3}{2}}}+\|v \times u\|_{\dot{B}_{2,1}^{\frac{3}{2}}} \lesssim\|u\|_{\dot{B}_{2,1}^{\frac{3}{2}}}^{2}+\left(\|B\|_{\dot{B}_{2,1}^{\frac{3}{2}}}+\|u\|_{\dot{B}_{2,1}^{\frac{3}{2}}}\right)\|v\|_{\dot{B}_{2,1}^{\frac{3}{2}}} \\
& \lesssim\left\|u^{L}\right\|_{\dot{B}_{2,1}^{\frac{3}{2}}}^{2}+\left\|B^{L}\right\|_{\dot{B}_{2,1}^{\frac{3}{2}}}^{2}+\left\|v^{L}\right\|_{\dot{B}_{2,1}^{\frac{1}{2}}}\left\|v^{L}\right\|_{\dot{B}_{2,1}^{\frac{5}{2}}} \\
& +\|\widetilde{u}\|_{\dot{B}_{2,1}^{\frac{1}{2}}}\|\widetilde{u}\|_{\dot{B}_{2,1}^{\frac{5}{2}}}+\|\widetilde{B}\|_{\dot{B}_{2,1}^{\frac{1}{2}}}\|\widetilde{B}\|_{\dot{B}_{2,1}^{\frac{5}{2}}}+\|\widetilde{v}\|_{\dot{B}_{2,1}^{\frac{1}{2}}}\|\widetilde{v}\|_{\dot{B}_{2,1}^{\frac{5}{2}}} .
\end{aligned}
$$

Using that $B=\operatorname{curl}^{-1}(u-v)$ and that $\nabla \operatorname{curl}^{-1}$ is a self-map on $\dot{B}_{2,1}^{\frac{3}{2}}$ (see Proposition A.2 (vii)) yields

$$
\begin{aligned}
\|v \cdot \nabla B\|_{\dot{B}_{2,1}^{\frac{3}{2}}} & \lesssim\|v\|_{\dot{B}_{2,1}^{\frac{3}{2}}}\left\|\nabla \operatorname{curl}^{-1}(u-v)\right\|_{\dot{B}_{2,1}^{\frac{3}{2}}} \\
& \lesssim\|v\|_{\dot{B}_{2,1}^{\frac{3}{2}}}^{2}+\|u\|_{\dot{B}_{2,1}^{\frac{3}{2}}}^{2} \\
& \lesssim\left\|u^{L}\right\|_{\dot{B}_{2,1}^{\frac{3}{2}}}^{2}+\left\|v^{L}\right\|_{\dot{B}_{2,1}^{\frac{1}{2}}}\left\|v^{L}\right\|_{\dot{B}_{2,1}^{\frac{5}{2}}}+\|\widetilde{u}\|_{\dot{B}_{2,1}^{\frac{1}{2}}}\|\widetilde{u}\|_{\dot{B}_{2,1}^{\frac{5}{2}}}+\|\widetilde{v}\|_{\dot{B}_{2,1}^{\frac{1}{2}}}\|\widetilde{v}\|_{\dot{B}_{2,1}^{\frac{5}{2}}}
\end{aligned}
$$


and, using also (A.6),

$$
\begin{aligned}
\left\|\left(\nabla \times v^{L}\right) \times B\right\|_{\dot{B}_{2,1}^{\frac{3}{2}}} & \lesssim\left\|\nabla \times v^{L}\right\|_{\dot{B}_{2,1}^{\frac{3}{2}}}\|B\|_{\dot{B}_{2,1}^{\frac{3}{2}}} \\
& \lesssim\left\|v^{L}\right\|_{\dot{B}_{2,1}^{\frac{5}{2}}}\left\|\operatorname{curl}^{-1}(u-v)\right\|_{\dot{B}_{2,1}^{\frac{3}{2}}} \\
& \lesssim\left\|v^{L}\right\|_{\dot{B}_{2,1}^{\frac{5}{2}}}\left(\left\|u^{L}\right\|_{\dot{B}_{2,1}^{\frac{1}{2}}}+\left\|v^{L}\right\|_{\dot{B}_{2,1}^{\frac{1}{2}}}+\|\widetilde{u}\|_{\dot{B}_{2,1}^{\frac{1}{2}}}+\|\widetilde{v}\|_{\dot{B}_{2,1}^{\frac{1}{2}}}\right) .
\end{aligned}
$$

From the estimate (A.8) with $s=3 / 2$ and the embedding $\dot{B}_{2,1}^{\frac{3}{2}} \hookrightarrow L^{\infty}$, we get

$$
\sum_{j} 2^{\frac{3 j}{2}}\left\|\left[\dot{\Delta}_{j}, b\right] a\right\|_{L^{2}} \lesssim\|\nabla b\|_{\dot{B}_{2,1}^{\frac{3}{2}}}\|a\|_{\dot{B}_{2,1}^{\frac{1}{2}}}
$$

whence

$$
\begin{aligned}
\sum_{j} 2^{\frac{3 j}{2}}\left\|\left[\dot{\Delta}_{j}, B \times\right](\nabla \times \widetilde{v})\right\|_{L^{2}} & \lesssim\|v-u\|_{\dot{B}_{2,1}^{\frac{3}{2}}}^{2}+\|\widetilde{v}\|_{\dot{B}_{2,1}^{\frac{3}{2}}}^{2} \\
& \lesssim\left\|u^{L}\right\|_{\dot{B}_{2,1}^{\frac{3}{2}}}^{2}+\left\|v^{L}\right\|_{\dot{B}_{2,1}^{\frac{1}{2}}}\left\|v^{L}\right\|_{\dot{B}_{2,1}^{\frac{5}{2}}} \\
& \quad+\|\widetilde{u}\|_{\dot{B}_{2,1}^{\frac{1}{2}}}^{\frac{1}{2}}\|\widetilde{u}\|_{\dot{B}_{2,1}^{\frac{5}{2}}}+\|\widetilde{v}\|_{\dot{B}_{2,1}^{\frac{1}{2}}}\|\widetilde{v}\|_{\dot{B}_{2,1}^{\frac{5}{2}}}
\end{aligned}
$$

Plugging the above estimates into the right-hand side of (4.7) and using (4.4), we end up with

$$
X(t)+C_{1} \int_{0}^{t} D(\tau) d \tau \leq C \int_{0}^{t} X(\tau) D(\tau) d \tau+C \int_{0}^{t}\left(c_{1}(\tau) X(\tau)+c_{2}(\tau)\right) d \tau,
$$

where $c_{1}$ and $c_{2}$ have been defined in the proposition,

$$
\begin{aligned}
X(t) & :=\|\widetilde{u}(t)\|_{\dot{B}_{2,1}^{\frac{1}{2}}}+\|\widetilde{B}(t)\|_{\dot{B}_{2,1}^{\frac{1}{2}}}+\|\widetilde{v}(t)\|_{\dot{B}_{2,1}^{\frac{1}{2}}} \\
\text { and } \quad D(t) & :=\|\widetilde{u}(t)\|_{\dot{B}_{2,1}^{\frac{5}{2}}}+\|\widetilde{B}(t)\|_{\dot{B}_{2,1}^{\frac{5}{2}}}+\|\widetilde{v}(t)\|_{\dot{B}_{2,1}^{\frac{5}{2}}} .
\end{aligned}
$$

Note that whenever

$$
2 C \sup _{\tau \in[0, t]} X(\tau) \leq C_{1}
$$

Inequality (4.9) combined with Gronwall lemma implies that

$$
X(t)+\frac{C_{1}}{2} \int_{0}^{t} D(\tau) d \tau \leq C \int_{0}^{t} c_{2}(\tau) e^{C \int_{\tau}^{t} c_{1}\left(\tau^{\prime}\right) d \tau^{\prime}} d \tau .
$$

Now, if Condition (4.1) is satisfied with $\kappa:=C_{1} / 2 C^{2}$, then the fact that the lefthand side of (4.9) is a continuous function on $[0, T]$ that vanishes at 0 combined with a standard bootstrap argument allows to prove that (4.10) and thus (4.1) is satisfied. Renaming the constants completes the proof of the proposition.

Second step: Constructing approximate solutions. It is based on Friedrichs' method : consider the spectral cut-off operator $\mathbb{E}_{n}$ defined by

$$
\mathcal{F}\left(\mathbb{E}_{n} f\right)(\xi)=1_{\left\{n^{-1} \leq|\xi| \leq n\right\}}(\xi) \mathcal{F}(f)(\xi) .
$$

We want to solve the following truncated system:

$$
\left\{\begin{array}{l}
\partial_{t} u-\Delta u=\mathbb{E}_{n} \mathcal{P}\left(\mathbb{E}_{n} B \cdot \mathbb{E}_{n} \nabla B-\mathbb{E}_{n} u \cdot \nabla \mathbb{E}_{n} u\right), \\
\partial_{t} B-\Delta B=\nabla \times \mathbb{E}_{n}\left(\mathbb{E}_{n}(u-\nabla \times B) \times \mathbb{E}_{n} B\right),
\end{array}\right.
$$

supplemented with initial data $\left(\mathbb{E}_{n} u_{0}, \mathbb{E}_{n} B_{0}\right)$. 
We need the following obvious lemma:

Lemma 4.2. Let $s \in \mathbb{R}$ and $k \geq 0$. Let $f \in \dot{B}_{2,1}^{s}$. Then, for all $n \geq 1$, we have

$$
\begin{gathered}
\left\|\mathbb{E}_{n} f\right\|_{\dot{B}_{2,1}^{s+k}} \lesssim n^{k}\|f\|_{\dot{B}_{2,1}^{s}}, \\
\lim _{n \rightarrow \infty}\left\|\mathbb{E}_{n} f-f\right\|_{\dot{B}_{2,1}^{s}}=0, \\
\left\|\mathbb{E}_{n} f-f\right\|_{\dot{B}_{2,1}^{s}} \lesssim \frac{1}{n^{k}}\|f\|_{\dot{B}_{2,1}^{s+k}} .
\end{gathered}
$$

We claim that (4.12) is an ODE in the Banach space $L^{2}\left(\mathbb{R}^{3} ; \mathbb{R}^{3} \times \mathbb{R}^{3}\right)$ for which the standard Cauchy-Lipschitz theorem applies. Indeed, the above lemma ensures that $\mathbb{E}_{n}$ maps $L^{2}$ to all Besov spaces, and that the right-hand side of (4.12) is a continuous bilinear map from $L^{2}\left(\mathbb{R}^{3} ; \mathbb{R}^{3} \times \mathbb{R}^{3}\right)$ to itself. We thus deduce that (4.12) admits a unique maximal solution $\left(u^{n}, B^{n}\right) \in \mathcal{C}^{1}\left(\left[0, T^{n}\right) ; L^{2}\left(\mathbb{R}^{3} ; \mathbb{R}^{3} \times \mathbb{R}^{3}\right)\right)$. Furthermore, as $\mathbb{E}_{n}^{2}=\mathbb{E}_{n}$, uniqueness implies $\mathbb{E}_{n} u^{n}=u^{n}$ and $\mathbb{E}_{n} B^{n}=B^{n}$, and we clearly have $\operatorname{div} u^{n}=\operatorname{div} B^{n}=0$. Being spectrally supported in the annulus $\left\{n^{-1} \leq|\xi| \leq n\right\}$, one can also deduce that the solution belongs to $\mathcal{C}^{1}\left(\left[0, T^{n}\right) ; \dot{B}_{2,1}^{s}\right)$ for all $s \in \mathbb{R}$. Hence, setting $J^{n}:=\nabla \times B^{n}$ and $v^{n}:=u^{n}-J^{n}$, we see that $u^{n}, B^{n}$ and $v^{n}$ belong to the space $E_{2}(T)$ for all $T<T^{n}$ and fulfill:

$$
\left\{\begin{array}{r}
\partial_{t} u^{n}-\Delta u^{n}=\mathbb{E}_{n} \mathcal{P}\left(B^{n} \cdot \nabla B^{n}-u^{n} \cdot \nabla u^{n}\right) \\
\partial_{t} B^{n}-\Delta B^{n}=\nabla \times \mathbb{E}_{n}\left(v^{n} \times B^{n}\right), \\
\partial_{t} v^{n}-\Delta v^{n}=\mathbb{E}_{n} \mathcal{P}\left(B^{n} \cdot \nabla B^{n}-u^{n} \cdot \nabla u^{n}-\nabla \times\left(\left(\nabla \times v^{n}\right) \times B^{n}\right)\right. \\
\left.\quad+\nabla \times\left(v^{n} \times u^{n}\right)+2 \nabla \times\left(v^{n} \cdot \nabla B^{n}\right)\right) .
\end{array}\right.
$$

Third step: uniform estimates

We want to apply Proposition 4.1 to our approximate solution $\left(u^{n}, B^{n}, v^{n}\right)$. The key point is that since $\mathbb{E}_{n}$ is an $L^{2}$ orthogonal projector, it has no effect on the energy estimates. We claim that $T^{n}$ may be bounded from below by the supremum $T$ of all the times satisfying (4.1), and that $\left(u^{n}, B^{n}, v^{n}\right)_{n \geq 1}$ is bounded in $E_{2}(T)$. To prove our claim, , we split $\left(u^{n}, B^{n}, v^{n}\right)$ into

$$
\left(u^{n}, B^{n}, v^{n}\right)=\left(u^{n, L}, B^{n, L}, v^{n, L}\right)+\left(\widetilde{u}^{n}, \widetilde{B}^{n}, \widetilde{v}^{n}\right),
$$

where

$$
u^{n, L}:=\mathbb{E}_{n} e^{t \Delta} u_{0}, \quad B^{n, L}:=\mathbb{E}_{n} e^{t \Delta} B_{0} \quad \text { and } \quad v^{n, L}:=\mathbb{E}_{n} e^{t \Delta} v_{0} .
$$

Since $\mathbb{E}_{n}$ maps any Besov space $\dot{B}_{2,1}^{s}$ to itself with norm 1, Condition (4.1) may be made independent of $n$ and thus, so does the corresponding time $T$. Now, as $\left(\widetilde{u}^{n}, \widetilde{B}^{n}, \widetilde{v}^{n}\right)$ is spectrally supported in $\left\{\xi \in \mathbb{R}^{3}\left|n^{-1} \leq\right| \xi \mid \leq n\right\}$, the estimate (4.2) ensures that it belongs to $L^{\infty}\left([0, T] ; L^{2}\left(\mathbb{R}^{3}\right)\right)$. So, finally, the standard continuation criterion for ordinary differential equations implies that $T^{n}$ is greater than any time $T$ satisfying (4.1) and that we have, for all $n \geq 1$,

$$
\begin{aligned}
& \left\|\left(\widetilde{u}^{n}, \widetilde{B}^{n}, \widetilde{v}^{n}\right)\right\|_{L_{T}^{\infty}\left(\dot{B}_{2,1}^{\frac{1}{2}}\right)}+C_{1}\left\|\left(\widetilde{u}^{n}, \widetilde{B}^{n}, \widetilde{v}^{n}\right)\right\|_{L_{T}^{1}\left(\dot{B}_{2,1}^{\frac{5}{2}}\right)} \leq C \kappa \text { and } \\
& \left\|\left(u^{n}, B^{n}, v^{n}\right)\right\|_{L_{T}^{\infty}\left(\dot{B}_{2,1}^{\frac{1}{2}}\right)}+C_{1}\left\|\left(u^{n}, B^{n}, v^{n}\right)\right\|_{L_{T}^{1}\left(\dot{B}_{2,1}^{\frac{5}{2}}\right)} \leq\left\|\left(u_{0}, B_{0}, v_{0}\right)\right\|_{\dot{B}_{2,1}^{\frac{1}{2}}}+C \kappa .
\end{aligned}
$$

\section{Fourth step: existence of a solution}


We claim that, up to an extraction, the sequence $\left(u^{n}, B^{n}, v^{n}\right)_{n \in \mathbb{N}}$ converges in $\mathcal{D}^{\prime}\left(\mathbb{R}^{+} \times \mathbb{R}^{3}\right)$ to a solution $(u, B, v)$ of (2.14) supplemented with data $\left(u_{0}, B_{0}, v_{0}\right)$ having the desired regularity properties. The definition of $\mathbb{E}_{n}$ entails that

$$
\left(\mathbb{E}_{n} u_{0}, \mathbb{E}_{n} B_{0}, \mathbb{E}_{n} v_{0}\right) \rightarrow\left(u_{0}, B_{0}, v_{0}\right) \quad \text { in } \dot{B}_{2,1}^{\frac{1}{2}},
$$

and Proposition A.4 thus ensures that $\left(u^{n, L}, B^{n, L}, v^{n, L}\right) \rightarrow\left(u^{L}, B^{L}, v^{L}\right)$ in $E_{2}(T)$.

Proving the convergence of $\left(\widetilde{u}^{n}, \widetilde{B}^{n}, \widetilde{v}^{n}\right)$ will be achieved from compactness arguments : we shall exhibit uniform bounds in suitable spaces for $\left(\partial_{t} u^{n}, \partial_{t} B^{n}, \partial_{t} v^{n}\right)_{n \in \mathbb{N}}$ so as to glean some Hölder regularity with respect to the time variable. Then, combining with compact embedding will enable us to apply Ascoli's theorem and to get the existence of a limit $(u, B, v)$ for a subsequence. Furthermore, the uniform bounds of the previous steps provide us with additional regularity and convergence properties so that we will be able to pass to the limit in (4.16). Let us start with a lemma.

Lemma 4.3. Sequence $\left(\widetilde{u}^{n}, \widetilde{B}^{n}, \widetilde{v}^{n}\right)_{n \geq 1}$ is bounded in $\mathcal{C}^{\frac{1}{2}}\left([0, T] ; \dot{B}_{2,1}^{-\frac{1}{2}}\right)$.

Proof. Observe that $\left(\widetilde{u}^{n}, \widetilde{B}^{n}, \widetilde{v}^{n}\right)$ satisfies

$$
\left\{\begin{aligned}
& \partial_{t} \widetilde{u}^{n}=\Delta \widetilde{u}^{n}+\mathbb{E}_{n} \mathcal{P}\left(B^{n} \cdot \nabla B^{n}-u^{n} \cdot \nabla u^{n}\right), \\
& \partial_{t} \widetilde{B}^{n}=\Delta \widetilde{B}^{n}+\nabla \times \mathbb{E}_{n}\left(v^{n} \times B^{n}\right), \\
& \partial_{t} \widetilde{v}^{n}=\Delta \widetilde{v}^{n}+\mathbb{E}_{n} \mathcal{P}\left(B^{n} \cdot \nabla B^{n}-u^{n} \cdot \nabla u^{n}-\nabla \times\left(\left(\nabla \times v^{n}\right) \times B^{n}\right)\right. \\
&\left.\quad+\nabla \times\left(v^{n} \times u^{n}\right)+2 \nabla \times\left(v^{n} \cdot \nabla B^{n}\right)\right) .
\end{aligned}\right.
$$

According to the uniform bounds (4.17), (4.18) and to the product laws:

$$
\|a b\|_{\dot{B}_{2,1}^{-\frac{1}{2}}} \lesssim\|a\|_{\dot{B}_{2,1}^{\frac{1}{2}}}\|b\|_{\dot{B}_{2,1}^{\frac{1}{2}}} \text { and }\|a b\|_{\dot{B}_{2,1}^{\frac{1}{2}}} \lesssim\|a\|_{\dot{B}_{2,1}^{\frac{1}{2},}}\|b\|_{\dot{B}_{2,1}^{\frac{3}{2}}},
$$

the right-hand side of (4.19) is uniformly bounded in $L_{T}^{2}\left(\dot{B}_{2,1}^{-\frac{1}{2}}\right)$. Since $\widetilde{u}^{n}(0)=$ $\widetilde{B}^{n}(0)=\widetilde{b}^{n}(0)=0$, applying Hölder inequality completes the proof.

We can now come to the proof of the existence of a solution. Let $\left(\phi_{j}\right)_{j \in \mathbb{N}}$ be a sequence of $\mathcal{C}_{0}^{\infty}\left(\mathbb{R}^{3}\right)$ cut-off functions supported in the ball $B(0, j+1)$ of $\mathbb{R}^{3}$ and equal to 1 in a neighborhood of $B(0, j)$. Lemma 4.3 tells us that $\left(\widetilde{u}^{n}, \widetilde{B}^{n}, \widetilde{v}^{n}\right)_{n \geq 1}$ is uniformly equicontinuous in the space $\mathcal{C}\left([0, T] ; \dot{B}_{2,1}^{-\frac{1}{2}}\right)$ and (4.17) ensures that it is bounded in $L^{\infty}\left([0, T] ; \dot{B}_{2,1}^{\frac{1}{2}}\right)$. Using the fact that the application $u \mapsto \phi_{j} u$ is compact from $\dot{B}_{2,1}^{\frac{1}{2}}$ into $\dot{B}_{2,1}^{-\frac{1}{2}}$, combining Ascoli's theorem and Cantor's diagonal process ensures that there exists some triplet $(\widetilde{u}, \widetilde{B}, \widetilde{v})$ such that for all $j \in \mathbb{N}$,

$$
\left(\phi_{j} \widetilde{u}^{n}, \phi_{j} \widetilde{B}^{n}, \phi_{j} \widetilde{v}^{n}\right) \rightarrow\left(\phi_{j} \widetilde{u}, \phi_{j} \widetilde{B}, \phi_{j} \widetilde{v}\right) \quad \text { in } \quad \mathcal{C}\left([0, T] ; \dot{B}_{2,1}^{-\frac{1}{2}}\right) .
$$

This obviously entails that $\left(\widetilde{u}^{n}, \widetilde{B}^{n}, \widetilde{v}^{n}\right)$ tends to $(\widetilde{u}, \widetilde{B}, \widetilde{v})$ in $\mathcal{D}^{\prime}\left(\mathbb{R}^{+} \times \mathbb{R}^{3}\right)$.

Coming back to the uniform estimates of third step and using the argument of 3 , p. 443] to justify that there is no time concentration, we get that $(\widetilde{u}, \widetilde{B}, \widetilde{v})$ belongs to $L^{\infty}\left(0, T ; \dot{B}_{2,1}^{\frac{1}{2}}\right) \cap L^{1}\left(0, T ; \dot{B}_{2,1}^{\frac{5}{2}}\right)$ and to $\mathcal{C}^{\frac{1}{2}}\left([0, T] ; \dot{B}_{2,1}^{-\frac{1}{2}}\right)$.

Let us now prove that $(u, B, v):=\left(u^{L}+\widetilde{u}, B^{L}+\widetilde{B}, v^{L}+\widetilde{v}\right)$ solves (2.14). The only problem is to pass to the limit in the non-linear terms. By way of example, let 
us explain how to handle the term $\mathbb{E}_{n} \mathcal{P} \nabla \times\left(\left(\nabla \times v^{n}\right) \times B^{n}\right)$ in (4.16) (actually, $\mathcal{P}$ may be omitted as a curl is divergence free). Let $\theta \in \mathcal{C}_{0}^{\infty}\left(\mathbb{R}^{+} \times \mathbb{R}^{3} ; \mathbb{R}^{3}\right)$ and $j \in \mathbb{N}$ be such that $\operatorname{Supp} \theta \subset[0, j] \times B(0, j)$. We use the decomposition

$$
\begin{aligned}
&\left\langle\nabla \times \mathbb{E}_{n}\left(\left(\nabla \times v^{n}\right) \times B^{n}\right), \theta\right\rangle-\langle\nabla \times((\nabla \times v) \times B), \theta\rangle \\
&=\left\langle\left(\nabla \times v^{n}\right) \times \phi_{j}\left(B^{n}-B\right), \nabla \times \mathbb{E}_{n} \theta\right\rangle+\left\langle\left(\nabla \times \phi_{j}\left(v^{n}-v\right)\right) \times B, \nabla \times \mathbb{E}_{n} \theta\right\rangle \\
&+\left\langle\mathbb{E}_{n}((\nabla \times v) \times B)-(\nabla \times v) \times B, \nabla \times \theta\right\rangle .
\end{aligned}
$$

As $\nabla \times v^{n}$ is uniformly bounded in $L_{T}^{1}\left(\dot{B}_{2,1}^{\frac{3}{2}}\right)$ and $\phi_{j} B^{n}$ tends to $\phi_{j} B$ in $L_{T}^{\infty}\left(\dot{B}_{2,1}^{\frac{1}{2}}\right)$, the first term tends to 0. According to the uniform estimates (4.18) and (4.20), $\nabla \times \phi_{j}\left(v^{n}-v\right)$ tends to 0 in $L_{T}^{1}\left(\dot{B}_{2,1}^{\frac{1}{2}}\right)$ so that the second term tends to 0 as well. Finally, thanks to (4.14), the third term tends to 0 .

The other non-linear terms can be treated similarly, and the continuity of $(u, B, v)$ stems from Proposition A.4 since the right-hand side of (2.14) belongs to $L_{T}^{1}\left(\dot{B}_{2,1}^{\frac{1}{2}}\right)$.

\section{Fifth step: uniqueness}

Let $\left(u_{1}, B_{1}\right)$ and $\left(u_{2}, B_{2}\right)$ be two solutions of the Hall-MHD system on $[0, T] \times \mathbb{R}^{3}$, with the same initial data, and such that $\left(u_{i}, B_{i}, v_{i}\right) \in E_{2}(T)$ for $i=1,2$. Then, the difference $(\delta u, \delta B, \delta v):=\left(u_{1}-u_{2}, B_{1}-B_{2}, v_{1}-v_{2}\right)$ is in $E_{2}(T)$ and satisfies

$$
\left\{\begin{array}{l}
\partial_{t} \delta u-\Delta \delta u:=R_{1}, \\
\partial_{t} \delta B-\Delta \delta B:=R_{2}, \\
\partial_{t} \delta v-\Delta \delta v:=R_{1}+R_{3}+R_{4}+R_{5},
\end{array}\right.
$$

where

$$
\begin{aligned}
& R_{1}:=\mathcal{P}\left(B_{1} \cdot \nabla \delta B+\delta B \cdot \nabla B_{2}-u_{1} \cdot \nabla \delta u-\delta u \cdot \nabla u_{2}\right) \\
& R_{2}:=\nabla \times\left(v_{1} \times \delta B+\delta v \times B_{2}\right), \\
& R_{3}:=-\nabla \times\left(\left(\nabla \times v_{1}\right) \times \delta B+(\nabla \times \delta v) \times B_{2}\right), \\
& R_{4}:=\nabla \times\left(v_{1} \times \delta u+\delta v \times u_{2}\right), \\
& R_{5}:=2 \nabla \times\left(v_{1} \cdot \nabla \delta B+\delta v \cdot \nabla B_{2}\right) .
\end{aligned}
$$

Hence, arguing as in the first step of the proof gives for all $t \in[0, T]$,

$$
\begin{aligned}
\|(\delta u, \delta B, \delta v)(t)\|_{\dot{B}_{2,1}^{\frac{1}{2}}}+\int_{0}^{t}\|(\delta u, \delta B, \delta v)\|_{\dot{B}_{2,1}^{\frac{5}{2}}} d \tau \lesssim \int_{0}^{t}\left(\left\|\left(R_{1}, R_{2}, R_{4}, R_{5}\right)\right\|_{\dot{B}_{2,1}^{\frac{1}{2}}}\right. \\
\left.+\left\|\nabla \times\left(\left(\nabla \times v_{1}\right) \times \delta B\right)\right\|_{\dot{B}_{2,1}^{\frac{1}{2}}}+\sum_{j \in \mathbb{Z}} 2^{\frac{3 j}{2}}\left\|\left[\dot{\Delta}_{j}, B_{2} \times\right](\nabla \times \delta v)\right\|_{L^{2}}\right) d \tau .
\end{aligned}
$$

Putting together the product laws (A.6) and the commutator estimate (4.8) yields

$$
\begin{aligned}
\left\|R_{1}\right\|_{\dot{B}_{2,1}^{\frac{1}{2}}} & \lesssim\left\|\left(u_{1}, B_{1}, u_{2}, B_{2}\right)\right\|_{\dot{B}_{2,1}^{\frac{3}{2}}}\|(\delta u, \delta B)\|_{\dot{B}_{2,1}^{\frac{3}{2}}} \\
\left\|R_{2}\right\|_{\dot{B}_{2,1}^{\frac{1}{2}}} & \lesssim\left\|\left(B_{2}, v_{1}\right)\right\|_{\dot{B}_{2,1}^{\frac{3}{2}}}\|(\delta B, \delta v)\|_{\dot{B}_{2,1}^{\frac{3}{2}}}, \\
\left\|R_{4}\right\|_{\dot{B}_{2,1}^{\frac{1}{2}}} & \lesssim\left\|\left(u_{2}, v_{1}\right)\right\|_{\dot{B}_{2,1}^{\frac{3}{2}}}\|(\delta u, \delta v)\|_{\dot{B}_{2,1}^{\frac{3}{2}}} \\
\left\|R_{5}\right\|_{\dot{B}_{2,1}^{\frac{1}{2}}} & \lesssim\left\|\left(\nabla B_{2}, v_{1}\right)\right\|_{\dot{B}_{2,1}^{\frac{3}{2}}} \|\left(\nabla \delta B, \delta v \|_{\dot{B}_{2,1}^{\frac{3}{2}}}\right. \\
& \lesssim\left\|\left(u_{2}, v_{1}, v_{2}\right)\right\|_{\dot{B}_{2,1}^{\frac{3}{2}}}\|(\delta u, \delta v)\|_{\dot{B}_{2,1}^{\frac{3}{2}}}
\end{aligned}
$$


and

$$
\begin{aligned}
\left\|\nabla \times\left(\left(\nabla \times v_{1}\right) \times \delta B\right)\right\|_{\dot{B}_{2,1}^{\frac{1}{2}}} & \lesssim\left\|\nabla \times v_{1}\right\|_{\dot{B}_{2,1}^{\frac{3}{2}}}\|\delta B\|_{\dot{B}_{2,1}^{\frac{3}{2}}} \\
& \lesssim\left\|v_{1}\right\|_{\dot{B}_{2,1}^{\frac{5}{2}}}\|(\delta u, \delta v)\|_{\dot{B}_{2,1}^{\frac{1}{2}}}
\end{aligned}
$$

$$
\begin{aligned}
\sum_{j \in \mathbb{Z}} 2^{\frac{3 j}{2}}\left\|\left[\dot{\Delta}_{j}, B_{2} \times\right](\nabla \times \delta v)\right\|_{L^{2}} & \lesssim\left\|\nabla B_{2}\right\|_{\dot{B}_{2,1}^{\frac{3}{2}}}\|\nabla \times \delta v\|_{\dot{B}_{2,1}^{\frac{1}{2}}} \\
& \lesssim\left\|\left(u_{2}, v_{2}\right)\right\|_{\dot{B}_{2,1}^{\frac{3}{2}}}\|\delta v\|_{\dot{B}_{2,1}^{\frac{3}{2}}} \cdot
\end{aligned}
$$

Hence, by interpolation and Young's inequality, Inequality (4.22) becomes

$$
\|(\delta u, \delta B, \delta v)(t)\|_{\dot{B}_{2,1}^{\frac{1}{2}}}+\int_{0}^{t}\|(\delta u, \delta B, \delta v)(\tau)\|_{\dot{B}_{2,1}^{\frac{5}{2}}} d \tau \leq \int_{0}^{t} Z(\tau)\|(\delta u, \delta B, \delta v)(\tau)\|_{\dot{B}_{2,1}^{\frac{1}{2}}} d \tau
$$

with $Z(t):=C\left(\left\|\left(u_{1}, u_{2}, B_{1}, B_{2}, v_{1}, v_{2}\right)\right\|_{\dot{B}_{2,1}^{\frac{3}{2}}}^{2}+\left\|v_{1}\right\|_{\dot{B}_{2,1}^{\frac{5}{2}}}\right) \cdot$

Thus, Gronwall lemma and our assumptions on the solutions ensure that

$$
(\delta u, \delta B, \delta v) \equiv 0 \quad \text { on } \quad[0, T]
$$

\section{Sixth step: Blow-up criterion}

Let us assume that we are given a solution $(u, B)$ on some finite time interval $\left[0, T^{*}\right)$ fulfilling the regularity properties listed in Theorem 2.2 for all $t<T^{*}$. Then, applying the method of the first step to (2.14) yields for all $t<T^{*}$,

$$
\begin{aligned}
& \|(u, B, v)(t)\|_{\dot{B}_{2,1}^{\frac{1}{2}}}+C_{1} \int_{0}^{t}\|(u, B, v)\|_{\dot{B}_{2,1}^{\frac{5}{2}}} d \tau \leq\|(u, B, v)(0)\|_{\dot{B}_{2,1}^{\frac{1}{2}}} \\
& +\int_{0}^{t}\left(\|B \cdot \nabla B\|_{\dot{B}_{2,1}^{\frac{1}{2}}}+\|u \cdot \nabla u\|_{\dot{B}_{2,1}^{\frac{1}{2}}}+\left(\|v \times B\|_{\dot{B}_{2,1}^{\frac{3}{2}}}+\|v \times u\|_{\dot{B}_{2,1}^{\frac{3}{2}}}\right.\right. \\
& \left.\left.+\|v \cdot \nabla B\|_{\dot{B}_{2,1}^{\frac{3}{2}}}+\sum_{j} 2^{\frac{3 j}{2}}\left\|\left[\dot{\Delta}_{j}, B \times\right](\nabla \times v)\right\|_{L^{2}}\right)\right) d \tau .
\end{aligned}
$$

Using the tame estimates (A.5), the fact that $\dot{B}_{2,1}^{\frac{3}{2}}$ is an algebra embedded in $L^{\infty}$, interpolation inequalities and Young's inequality, we get for all $\eta>0$,

$$
\begin{aligned}
\|B \cdot \nabla B\|_{\dot{B}_{2,1}^{\frac{1}{2}}} & \leq C\|B \otimes B\|_{\dot{B}_{2,1}^{\frac{3}{2}}} \\
& \leq C\|B\|_{L^{\infty}}\|B\|_{\dot{B}_{2,1}^{\frac{3}{2}}} \\
& \leq \frac{C}{\eta}\|B\|_{L^{\infty}}^{2}\|B\|_{\dot{B}_{2,1}^{\frac{1}{2}}}+\eta\|B\|_{\dot{B}_{2,1}^{\frac{5}{2}}},
\end{aligned}
$$

and, similarly,

$$
\|u \cdot \nabla u\|_{\dot{B}_{2,1}^{\frac{1}{2}}} \leq \frac{C}{\eta}\|u\|_{L^{\infty}}^{2}\|u\|_{\dot{B}_{2,1}^{\frac{1}{2}}}+\eta\|u\|_{\dot{B}_{2,1}^{\frac{5}{2}}} .
$$

We also have

$$
\begin{aligned}
\|v \times B\|_{\dot{B}_{2,1}^{\frac{3}{2}}} & \leq C\left(\|v\|_{L^{\infty}}\|B\|_{\dot{B}_{2,1}^{\frac{3}{2}}}+\|B\|_{L^{\infty}}\|v\|_{\dot{B}_{2,1}^{\frac{3}{2}}}\right) \\
& \leq \frac{C}{\eta}\|(B, v)\|_{L^{\infty}}^{2}\|(B, v)\|_{\dot{B}_{2,1}^{\frac{1}{2}}}+\eta\|(B, v)\|_{\dot{B}_{2,1}^{\frac{5}{2}}}, \\
\|v \times u\|_{\dot{B}_{2,1}^{\frac{3}{2}}} & \leq \frac{C}{\eta}\|(u, v)\|_{L^{\infty}}^{2}\|(u, v)\|_{\dot{B}_{2,1}^{\frac{1}{2}}}+\eta\|(u, v)\|_{\dot{B}_{2,1}^{\frac{5}{2}}},
\end{aligned}
$$




$$
\|v \cdot \nabla B\|_{\dot{B}_{2,1}^{\frac{3}{2}}} \leq \frac{C}{\eta}\|(\nabla B, v)\|_{L^{\infty}}^{2}\|(\nabla B, v)\|_{\dot{B}_{2,1}^{\frac{1}{2}}}+\eta\|(\nabla B, v)\|_{\dot{B}_{2,1}^{\frac{5}{2}}} .
$$

As, according to (A.8) with $s=3 / 2$ and to the fact that $\nabla: L^{\infty} \rightarrow \dot{B}_{\infty, \infty}^{-1}$, we have

$$
\sum_{j} 2^{\frac{3 j}{2}}\left\|\left[\dot{\Delta}_{j}, B \times\right](\nabla \times v)\right\|_{L^{2}} \leq C\left(\|\nabla B\|_{L^{\infty}}\|v\|_{\dot{B}_{2,1}^{\frac{3}{2}}}+\|v\|_{L^{\infty}\|\nabla B\|_{\dot{B}_{2,1}^{\frac{3}{2}}}}\right),
$$

that term may be bounded as $v \cdot \nabla B$.

Therefore, if we choose $\eta$ small enough, then (4.23) becomes:

$$
\begin{aligned}
&\|(u, B, v)(t)\|_{\dot{B}_{2,1}^{\frac{1}{2}}}+\frac{C_{1}}{2} \int_{0}^{t}\|(u, B, v)\|_{\dot{B}_{2,1}^{\frac{5}{2}}} d \tau \leq\|(u, B, v)(0)\|_{\dot{B}_{2,1}^{\frac{1}{2}}} \\
&+C \int_{0}^{t}\|(u, B, \nabla B)\|_{L^{\infty}}^{2}\|(u, B, v)\|_{\dot{B}_{2,1}^{\frac{1}{2}}} d \tau
\end{aligned}
$$

and Gronwall's inequality implies that for all $t \in\left[0, T^{*}\right)$,

$$
\begin{aligned}
\|(u, B, v)(t)\|_{\dot{B}_{2,1}^{\frac{1}{2}}}+\frac{C_{1}}{2} \int_{0}^{t}\|(u, B, v)\|_{\dot{B}_{2,1}^{\frac{5}{2}}} d \tau & \\
& \leq\|(u, B, v)(0)\|_{\dot{B}_{2,1}^{\frac{1}{2}}} \exp \left(C \int_{0}^{t}\|(u, B, \nabla B)\|_{L^{\infty}}^{2} d t\right) .
\end{aligned}
$$

Now, if one assumes that

$$
\int_{0}^{T^{*}}\|(u, B, \nabla B)(t)\|_{L^{\infty}}^{2} d t<\infty,
$$

then the above inequality ensures that $(u, B, v)$ belongs to $L^{\infty}\left(0, T^{*} ; \dot{B}_{2,1}^{\frac{1}{2}}\right)$ and one may conclude by classical arguments that the solution may be continued beyond $T^{*}$.

In order to prove the second blow-up criterion, one uses the following inequalities, based on (A.6) and interpolation inequalities:

$$
\begin{aligned}
& \|B \cdot \nabla B\|_{\dot{B}_{2,1}^{\frac{1}{2}}} \lesssim\|B\|_{\dot{B}_{2,1}^{\frac{1}{2}}}\|B\|_{\dot{B}_{2,1}^{\frac{5}{2}}}, \\
& \|u \cdot \nabla u\|_{\dot{B}_{2,1}^{\frac{1}{2}}} \lesssim\|u\|_{\dot{B}_{2,1}^{\frac{1}{2}}}\|u\|_{\dot{B}_{2,1}^{\frac{5}{2}}}, \\
& \|v \times B\|_{\dot{B}_{2,1}^{\frac{3}{2}}} \lesssim\|v\|_{\dot{B}_{2,1}^{\frac{3}{2}}}\|B\|_{\dot{B}_{2,1}^{\frac{3}{2}}} \\
& \lesssim\|v\|_{\dot{B}_{2,1}^{\frac{1}{2}}}\|v\|_{\dot{B}_{2,1}^{\frac{5}{2}}}+\|B\|_{\dot{B}_{2,1}^{\frac{1}{2}}}\|B\|_{\dot{B}_{2,1}^{\frac{5}{2}}}, \\
& \|v \times u\|_{\dot{B}_{2,1}^{\frac{3}{2}}} \lesssim\|v\|_{\dot{B}_{2,1}^{\frac{3}{2}}}\|u\|_{\dot{B}_{2,1}^{\frac{3}{2}}} \\
& \lesssim\|v\|_{\dot{B}_{2,1}^{\frac{1}{2}}}\|v\|_{\dot{B}_{2,1}^{\frac{5}{2}}}+\|u\|_{\dot{B}_{2,1}^{\frac{1}{2}}}\|u\|_{\dot{B}_{2,1}^{\frac{5}{2}}}, \\
& \|v \cdot \nabla B\|_{\dot{B}_{2,1}^{\frac{3}{2}}} \lesssim\|v\|_{\dot{B}_{2,1}^{\frac{3}{2}}}\|\nabla B\|_{\dot{B}_{2,1}^{\frac{3}{2}}} \\
& \lesssim\|v\|_{\dot{B}_{2,1}^{\frac{3}{2}}}^{2}+\|J\|_{\dot{B}_{2,1}^{\frac{3}{2}}}^{2} \\
& \lesssim\|v\|_{\dot{B}_{2,1}^{\frac{3}{2}}}^{2}+\|u\|_{\dot{B}_{2,1}^{\frac{3}{2}}}^{2} \\
& \lesssim\|v\|_{\dot{B}_{2,1}^{\frac{1}{2}}}\|v\|_{\dot{B}_{2,1}^{\frac{5}{2}}}+\|u\|_{\dot{B}_{2,1}^{\frac{1}{2}}}\|u\|_{\dot{B}_{2,1}^{\frac{5}{2}}}
\end{aligned}
$$


and by (4.8) and Proposition A.2 (iii) (vii),

$$
\begin{aligned}
\sum_{j} 2^{\frac{3 j}{2}}\left\|\left[\dot{\Delta}_{j}, B \times\right](\nabla \times v)\right\|_{L^{2}} & \lesssim\|\nabla B\|_{\dot{B}_{2,1}^{\frac{3}{2}}}\|v\|_{\dot{B}_{2,1}^{\frac{3}{2}}} \\
& \lesssim\|v\|_{\dot{B}_{2,1}^{\frac{1}{2}}}\|v\|_{\dot{B}_{2,1}^{\frac{5}{2}}}+\|u\|_{\dot{B}_{2,1}^{\frac{1}{2}}}\|u\|_{\dot{B}_{2,1}^{\frac{5}{2}}} .
\end{aligned}
$$

Plugging those estimates in (4.23), we find that

$$
\begin{aligned}
\|(u, B, v)(t)\|_{\dot{B}_{2,1}^{\frac{1}{2}}}+C_{1} \int_{0}^{t}\|(u, B, v)\|_{\dot{B}_{2,1}^{\frac{5}{2}}} d \tau \leq & \|(u, B, v)(0)\|_{\dot{B}_{2,1}^{\frac{1}{2}}} \\
& +\int_{0}^{t}\|(u, B, v)\|_{\dot{B}_{2,1}^{\frac{1}{2}}}\|(u, B, v)\|_{\dot{B}_{2,1}^{\frac{5}{2}}} d \tau .
\end{aligned}
$$

Hence, if

$$
\int_{0}^{T^{*}}\|(u, B, J)\|_{\dot{B}_{2,1}^{\frac{5}{2}}} d t<\infty
$$

then the solution may be continued beyond $T^{*}$.

For proving the last blow-up criterion, one can use that for $\rho \in(2, \infty]$, most of the terms of (4.23) may be bounded by means of Inequality A.7. The last commutator term may be bounded from (A.9) (without time integration) with $r=1$ and $s=3 / 2$ as follows:

$$
\sum_{j} 2^{\frac{3 j}{2}}\left\|\left[\dot{\Delta}_{j}, B \times\right](\nabla \times v)\right\|_{L^{2}} \lesssim\|\nabla B\|_{\dot{B}_{\infty, \infty}^{\frac{2}{\rho}-1}}\|v\|_{\dot{B}_{2,1}^{\frac{5}{2}-\frac{2}{\rho}}}+\|v\|_{\dot{B}_{\infty, \infty}^{\frac{2}{\rho}-1}}\|\nabla B\|_{\dot{B}_{2,1}^{\frac{5}{2}-\frac{2}{\rho}}} \cdot
$$

Since, by interpolation, we have

$$
\|Z\|_{\dot{B}_{2,1}^{\frac{5}{2}-\frac{2}{\rho}}} \lesssim\|Z\|_{\dot{B}_{2,1}^{\frac{1}{2}}}^{\frac{1}{\rho}}\|Z\|_{\dot{B}_{2,1}^{\frac{5}{2}}}^{\frac{1}{\rho^{\prime}}} \quad \text { with } \quad \frac{1}{\rho^{\prime}}=1-\frac{1}{\rho},
$$

using Young inequality and reverting to (4.23) yields

$$
\begin{aligned}
\|(u, B, v)(t)\|_{\dot{B}_{2,1}^{\frac{1}{2}}}+\int_{0}^{t}\|(u, B, v)\|_{\dot{B}_{2,1}^{\frac{5}{2}}} d \tau \leq & \|(u, B, v)(0)\|_{\dot{B}_{2,1}^{\frac{1}{2}}} \\
& +C \int_{0}^{t}\|(u, B, v)\|_{\dot{B}_{\infty, \infty}^{2}-1}^{\rho}\|(u, B, v)\|_{\dot{B}_{2,1}^{\frac{1}{2}}} d \tau .
\end{aligned}
$$

As before, one can conclude that if $T^{*}<\infty$ and (2.18) is fulfilled, then the solution may be continued beyond $T^{*}$. This completes the proof of the theorem.

\section{The Well-POSEDNESS THEORY IN SPACES $\dot{B}_{2, r}^{\frac{1}{2}}$ FOR General $r$}

Let us first prove the a priori estimates leading to global existence.

Proposition 5.1. Assume that $(u, B)$ is a smooth solution of the Hall-MHD system on $[0, T] \times \mathbb{R}^{3}$ with $h=\mu=\nu=1$. Let $v:=u-\nabla \times B$. There exists a universal constant $C$ such that for any $r \in[1, \infty]$, we have

$$
\|(u, B, v)\|_{E_{2, r}(T)} \leq C\left(\left\|\left(u_{0}, B_{0}, v_{0}\right)\right\|_{\dot{B}_{2, r}^{\frac{1}{2}}}+\|(u, B, v)\|_{E_{2, r}(T)}^{2}\right) .
$$

Proof. We argue as in the proof of Inequality (4.23), but take the $\ell^{r}(\mathbb{Z})$ norm instead of the $\ell^{1}(\mathbb{Z})$ norm. We get for all $t \in[0, T]$,

$$
\|(u, B, v)\|_{\widetilde{L}_{t}^{\infty}\left(\dot{B}_{2, r}^{\frac{1}{2}}\right)}+\|(u, B, v)\|_{\widetilde{L}_{t}^{1}\left(\dot{B}_{2, r}^{\frac{5}{2}}\right)} \lesssim\left\|\left(u_{0}, B_{0}, v_{0}\right)\right\|_{\dot{B}_{2, r}^{\frac{1}{2}}}+\|B \cdot \nabla B\|_{\widetilde{L}_{t}^{1}\left(\dot{B}_{2, r}^{\frac{1}{2}}\right)}
$$




$$
\begin{aligned}
& +\|u \cdot \nabla u\|_{\widetilde{L}_{t}^{1}\left(\dot{B}_{2, r}^{\frac{1}{2}}\right)}+\|v \cdot \nabla B\|_{\widetilde{L}_{t}^{1}\left(\dot{B}_{2, r}^{\frac{1}{2}}\right)}+\|B \cdot \nabla v\|_{\widetilde{L}_{t}^{1}\left(\dot{B}_{2, r}^{\frac{1}{2}}\right)}+\|v \cdot \nabla u\|_{\widetilde{L}_{t}^{1}\left(\dot{B}_{2, r}^{\frac{1}{2}}\right)} \\
& \quad+\|u \cdot \nabla v\|_{\widetilde{L}_{t}^{1}\left(\dot{B}_{2, r}^{\frac{1}{2}}\right)}+\|v \cdot \nabla B\|_{\widetilde{L}_{t}^{1}\left(\dot{B}_{2, r}^{\frac{3}{2}}\right)}+\left\|2^{\frac{3 j}{2}}\right\|\left[\dot{\Delta}_{j}, B \times\right](\nabla \times v)\left\|_{L_{t}^{1}\left(L^{2}\right)}\right\|_{\ell^{r}(\mathbb{Z})} .
\end{aligned}
$$

The first six nonlinear terms in the right-hand side may be bounded according to the following product law that is proved in Appendix:

$$
\|a b\|_{\widetilde{L}_{t}^{1}\left(\dot{B}_{2, r}^{\left.\frac{1}{2}\right)}\right)} \lesssim\|a\|_{\widetilde{L}_{t}^{4}\left(\dot{B}_{2, r}^{1}\right)}\|b\|_{\widetilde{L}_{t}^{\frac{4}{3}\left(\dot{B}_{2, r}^{1}\right)}} .
$$

The last but one term may be bounded as follows:

$$
\|v \cdot \nabla B\|_{\widetilde{L}_{t}^{1}\left(\dot{B}_{2, r}^{\left.\frac{3}{2}\right)}\right.} \lesssim\|v\|_{\widetilde{L}_{t}^{4}\left(\dot{B}_{2, r}^{1}\right)}\|\nabla B\|_{\widetilde{L}_{t}^{\frac{4}{3}\left(\dot{B}_{2, r}^{2}\right)}}+\|\nabla B\|_{\widetilde{L}_{t}^{4}\left(\dot{B}_{2, r}^{1}\right)}\|v\|_{\widetilde{L}_{t}^{\frac{4}{3}\left(\dot{B}_{2, r}^{2}\right)}} .
$$

Finally, in light of (A.9) with $b=B, a=\nabla \times v, s=3 / 2$ and $\rho=4$, and embedding, one discovers that the commutator term may be bounded exactly as $v \cdot \nabla B$.

Putting together all the above inequalities eventually yields for all $t \geq 0$,

$$
\|(u, B, v)\|_{E_{2, r}(t)} \lesssim\left\|\left(u_{0}, B_{0}, v_{0}\right)\right\|_{\dot{B}_{2, r}^{\frac{1}{2}}}+\|(u, B, v)\|_{\widetilde{L}_{t}^{\frac{4}{3}\left(\dot{B}_{2, r}^{2}\right)}}\|(u, B, v)\|_{\widetilde{L}_{t}^{4}\left(\dot{B}_{2, r}^{1}\right)} .
$$

Since one can prove by making use of Hölder inequality and interpolation that

$$
\|z\|_{\widetilde{L}_{t}^{\rho}\left(\dot{B}_{2, r}^{\left.\frac{1}{2}+\frac{2}{\rho}\right)}\right.} \leq\|z\|_{E_{2, r}(t)} \text { for all } \rho \in[1,+\infty]
$$

Inequality (5.4) implies (5.1).

In order to prove Theorem 2.3, we proceed as follows:

(1) smooth out the data and get a sequence $\left(u^{n}, B^{n}\right)_{n \in \mathbb{N}}$ of global smooth solutions to the Hall-MHD system;

(2) apply Proposition 5.1 to $\left(u^{n}, B^{n}\right)_{n \in \mathbb{N}}$ and obtain uniform estimates for $\left(u^{n}, B^{n}, v^{n}\right)_{n \in \mathbb{N}}$ in the space $E_{2, r}$

(3) use compactness to prove that $\left(u^{n}, B^{n}\right)_{n \in \mathbb{N}}$ converges, up to extraction, to a solution of the Hall-MHD system supplemented with initial data $\left(u_{0}, B_{0}\right)$;

(4) prove stability estimates in a larger space to get the uniqueness of the solution.

To proceed, let us smooth out the initial data as follow:

$$
u_{0}^{n}:=\left(\dot{S}_{n}-\dot{S}_{-n}\right) u_{0} \quad \text { and } \quad B_{0}^{n}:=\left(\dot{S}_{n}-\dot{S}_{-n}\right) B_{0} .
$$

Clearly, $u_{0}^{n}$ and $B_{0}^{n}$ belong to all Sobolev spaces, and we have for $z=u, B, v$ and all $n \in \mathbb{N}$,

$$
\forall j \in \mathbb{Z}, \quad\left\|\dot{\Delta}_{j} z_{0}^{n}\right\|_{L^{2}} \leq\left\|\dot{\Delta}_{j} z_{0}\right\|_{L^{2}} \quad \text { and } \quad\left\|z_{0}^{n}\right\|_{\dot{B}_{2, r}^{\frac{1}{2}}} \leq\left\|z_{0}\right\|_{\dot{B}_{2, r}^{\frac{1}{2}}} .
$$

Since in particular $\left(u_{0}^{n}, B_{0}^{n}, v_{0}^{n}\right)$ is in $\dot{B}_{2,1}^{\frac{1}{2}}$, Theorem 2.2 guarantees that the HallMHD system with data $\left(u_{0}^{n}, B_{0}^{n}\right)$ has a unique maximal solution on $\left[0, T^{n}\right)$ for some $T^{n}>0$, that belongs to $E_{2,1}(T)$ for all $T<T^{n}$. Now, take some positive real number $M$ to be chosen later on and define

$$
T_{n}:=\sup \left\{t \in\left[0, T^{n}\right),\left\|\left(u^{n}, B^{n}, v^{n}\right)\right\|_{E_{2, r}(t)} \leq M c\right\} .
$$

We are going to show first that $T_{n}=T^{n}$, then that $T^{n}=+\infty$.

\footnotetext{
${ }^{3}$ The reader may refer to the appendix for the definition of $\dot{S}_{j}$
} 
According to Proposition 5.1 and to (5.5), we have

$$
\left\|\left(u^{n}, B^{n}, v^{n}\right)\right\|_{E_{2, r}\left(T_{n}\right)} \leq C\left(\left\|\left(u_{0}, B_{0}, v_{0}\right)\right\|_{\dot{B}_{2, r}^{\frac{1}{2}}}+\left\|\left(u^{n}, B^{n}, v^{n}\right)\right\|_{E_{2, r}\left(T_{n}\right)}^{2}\right) .
$$

Hence, using the smallness condition on $\left(u_{0}, B_{0}, v_{0}\right)$ and the definition of $T_{n}$,

$$
\left\|\left(u^{n}, B^{n}, v^{n}\right)\right\|_{E_{2, r}\left(T_{n}\right)} \leq C c\left(1+M^{2} c\right) .
$$

If we take $M=2 C$, then $c$ so that $4 C^{2} c<1$, then we have

$$
\left\|\left(u^{n}, B^{n}, v^{n}\right)\right\|_{E_{2, r}\left(T_{n}\right)}<M c,
$$

and thus, by a classical continuity argument, $T_{n}=T^{n}$.

Now, using functional embedding and interpolation arguments, we discover that

$$
\left(\int_{0}^{T^{n}}\left\|\left(u^{n}, B^{n}, v^{n}\right)\right\|_{\dot{B}_{\infty, \infty}^{-\frac{1}{2}}}^{4} d t\right)^{\frac{1}{4}} \lesssim\left\|\left(u^{n}, B^{n}, v^{n}\right)\right\|_{\widetilde{L}_{T^{n}}^{4}\left(\dot{B}_{2, r}^{1}\right)} \lesssim\left\|\left(u^{n}, B^{n}, v^{n}\right)\right\|_{E_{2, r}\left(T^{n}\right)} .
$$

Hence, the continuation criterion (2.18) guarantees that, indeed, $T^{n}=+\infty$. This means that the solution is global and that, furthermore,

$$
\left\|\left(u^{n}, B^{n}, v^{n}\right)\right\|_{E_{2, r}} \leq M c \text { for all } n \in \mathbb{N} .
$$

At this stage, proving that $\left(u^{n}, B^{n}\right)_{n \in \mathbb{N}}$ converges (up to subsequence) to a global solution $(u, B)$ of the Hall-MHD system with data $\left(u_{0}, B_{0}\right)$ and $(u, B, v)$ in $E_{2, r}$ follows from the same arguments as in the previous section.

Let us finally prove the uniqueness part of the theorem. Suppose that $\left(u_{1}, B_{1}\right)$ and $\left(u_{2}, B_{2}\right)$ are two solutions of the Hall-MHD system on $[0, T] \times \mathbb{R}^{3}$ supplemented with the same initial data $\left(u_{0}, B_{0}\right)$ and such that

$$
\left(u_{i}, B_{i}, v_{i}\right) \in \widetilde{\mathcal{C}}\left([0, T] ; \dot{B}_{2, r}^{\frac{1}{2}}\right) \cap \widetilde{L}^{1}\left(0, T ; \dot{B}_{2, r}^{\frac{5}{2}}\right), \quad i=1,2 .
$$

In order to prove the uniqueness, we look at the difference $(\delta u, \delta B, \delta v)=\left(u_{1}-\right.$ $\left.u_{2}, B_{1}-B_{2}, v_{1}-v_{2}\right)$ as a solution of System (4.21). In contrast with the previous section however, we do not know how to estimate the difference in the space $E_{2, r}(T)$ since the term $\nabla \times\left(\left(\nabla \times v_{1}\right) \times \delta B\right)$ cannot be bounded in the space $\widetilde{L}_{T}^{1}\left(\dot{B}_{2, r}^{\frac{1}{2}}\right)$ from the norm of $v_{1}$ and $\delta B$ in $E_{2, r}(T)$ (this is due to the fact that the norm of $E_{2, r}(T)$ fails to control $\|\cdot\|_{L^{\infty}\left(0, T \times \mathbb{R}^{3}\right)}$ by a little if $\left.r>1\right)$.

For that reason, we shall accept to lose some regularity in the stability estimates and prove uniqueness in the space

$$
F_{2, r}(T):=\widetilde{L}_{T}^{\infty}\left(\dot{B}_{2, r}^{-\frac{1}{2}}\right)
$$

We need first to justify that $(\delta u, \delta B, \delta v)$ belongs to that space, though. According to Proposition A.4 it is enough to check that the terms $R_{1}$ to $R_{5}$ defined just below (4.21) belong to $\widetilde{L}_{T}^{1}\left(\dot{B}_{2, r}^{-\frac{1}{2}}\right)$. Now, from (5.2) and Holder inequality, we have

$$
\begin{aligned}
\left\|R_{1}\right\|_{\widetilde{L}_{T}^{1}\left(\dot{B}_{2, r}^{-\frac{1}{2}}\right)} & \lesssim T^{\frac{1}{2}}\left\|\left(u_{1}, B_{1}, u_{2}, B_{2}\right)\right\|_{\widetilde{L}_{T}^{4}\left(\dot{B}_{2, r}^{1}\right)}\|(\delta u, \delta B)\|_{\widetilde{L}_{T}^{4}\left(\dot{B}_{2, r}^{1}\right)}, \\
\left\|R_{2}\right\|_{\widetilde{L}_{T}^{1}\left(\dot{B}_{2, r}^{-\frac{1}{2}}\right)} & \lesssim T^{\frac{1}{2}}\left\|\left(B_{2}, v_{1}\right)\right\|_{\widetilde{L}_{T}^{4}\left(\dot{B}_{2, r}^{1}\right)}\|(\delta B, \delta v)\|_{\widetilde{L}_{T}^{4}\left(\dot{B}_{2, r}^{1}\right)}, \\
\left\|R_{3}\right\|_{\widetilde{L}_{T}^{1}\left(\dot{B}_{2, r}^{-\frac{1}{2}}\right)} & \lesssim\left\|\left(\nabla v_{1}, \nabla \delta v\right)\right\|_{\widetilde{L}_{T}^{\frac{4}{3}\left(\dot{B}_{2, r}^{1}\right)}}\left\|\left(\delta B, B_{2}\right)\right\|_{\widetilde{L}_{T}^{4}\left(\dot{B}_{2, r}^{1}\right)}, \\
\left\|R_{4}\right\|_{\widetilde{L}_{T}^{1}\left(\dot{B}_{2, r}^{-\frac{1}{2}}\right)} & \lesssim T^{\frac{1}{2}}\left\|\left(u_{2}, v_{1}\right)\right\|_{\widetilde{L}_{T}^{4}\left(\dot{B}_{2, r}^{1}\right)}\|(\delta u, \delta v)\|_{\widetilde{L}_{T}^{4}\left(\dot{B}_{2, r}^{1}\right)},
\end{aligned}
$$




$$
\left\|R_{5}\right\|_{\widetilde{L}_{T}^{1}\left(\dot{B}_{2, r}^{-\frac{1}{2}}\right)} \lesssim\left\|\left(\nabla B_{2}, \nabla \delta B\right)\right\|_{\widetilde{L}_{T}^{\frac{4}{3}\left(\dot{B}_{2, r}^{1}\right)}}\left\|\left(\delta v, v_{1}\right)\right\|_{\widetilde{L}_{T}^{4}\left(\dot{B}_{2, r}^{1}\right)} .
$$

Since the norm in $E_{2, r}(T)$ bounds the norm in $\widetilde{L}_{T}^{4}\left(\dot{B}_{2, r}^{1}\right) \cap \widetilde{L}_{T}^{\frac{4}{3}}\left(\dot{B}_{2, r}^{2}\right)$, one can indeed conclude that the terms $R_{1}$ to $R_{5}$ are in $\widetilde{L}_{T}^{1}\left(\dot{B}_{2, r}^{-\frac{1}{2}}\right)$.

Next, estimating $(\delta u, \delta B, \delta v)$ in $F_{2, r}(T)$ may be achieved by a slight modification of the beginning of the proof of Proposition 5.1. We get for all $t \in[0, T]$,

$$
\begin{aligned}
\|(\delta u, \delta B & , \delta v)\left\|_{F_{2, r}(t)} \lesssim\right\| B_{1} \cdot \nabla \delta B\left\|_{\widetilde{L}_{t}^{1}\left(\dot{B}_{2, r}^{-\frac{1}{2}}\right)}+\right\| \delta B \cdot \nabla B_{2} \|_{\widetilde{L}_{t}^{1}\left(\dot{B}_{2, r}^{-\frac{1}{2}}\right)} \\
& +\left\|u_{1} \cdot \nabla \delta u\right\|_{\widetilde{L}_{t}^{1}\left(\dot{B}_{2, r}^{-\frac{1}{2}}\right)}+\left\|\delta u \cdot \nabla u_{2}\right\|_{\widetilde{L}_{t}^{1}\left(\dot{B}_{2, r}^{-\frac{1}{2}}\right)}+\left\|v_{1} \cdot \nabla \delta B\right\|_{\widetilde{L}_{t}^{1}\left(\dot{B}_{2, r}^{-\frac{1}{2}}\right)} \\
& +\left\|\delta B \cdot \nabla v_{1}\right\|_{\left.\widetilde{L}_{t}^{1\left(\dot{B}_{2, r}^{-}\right.}-\frac{1}{2}\right)}+\left\|B_{2} \cdot \nabla \delta v\right\|_{\widetilde{L}_{t}^{1}\left(\dot{B}_{2, r}^{-\frac{1}{2}}\right)}+\left\|\delta v \cdot \nabla B_{2}\right\|_{\widetilde{L}_{t}^{1}\left(\dot{B}_{2, r}^{-\frac{1}{2}}\right)} \\
& +\left\|v_{1} \cdot \nabla \delta u\right\|_{\widetilde{L}_{t}^{1}\left(\dot{B}_{2, r}^{-\frac{1}{2}}\right)}+\left\|\delta u \cdot \nabla v_{1}\right\|_{\widetilde{L}_{t}^{1}\left(\dot{B}_{2, r}^{-\frac{1}{2}}\right)}+\left\|u_{2} \cdot \nabla \delta v\right\|_{\widetilde{L}_{t}^{1}\left(\dot{B}_{2, r}^{-\frac{1}{2}}\right)} \\
& +\left\|\delta v \cdot \nabla u_{2}\right\|_{\widetilde{L}_{t}^{1}\left(\dot{B}_{2, r}^{-\frac{1}{2}}\right)}+\left\|\left(\nabla \times v_{1}\right) \times \delta B\right\|_{\widetilde{L}_{t}^{1}\left(\dot{B}_{2, r}^{\frac{1}{2}}\right)}+\left\|v_{1} \cdot \nabla \delta B\right\|_{\widetilde{L}_{t}^{1}\left(\dot{B}_{2, r}^{\frac{1}{2}}\right)} \\
& +\left\|\delta v \cdot \nabla B_{2}\right\|_{\widetilde{L}_{t}^{1}\left(\dot{B}_{2, r}^{\frac{1}{2}}\right)}+\left\|2^{\frac{j}{2}}\right\|\left[\dot{\Delta}_{j}, B_{2} \times\right](\nabla \times \delta v)\left\|_{L_{t}^{1}\left(L^{2}\right)}\right\|_{\ell^{r}(\mathbb{Z})} \cdot
\end{aligned}
$$

Most of the terms on the right-hand side can be bounded by means of the following inequalities that are proved in appendix:

$$
\begin{aligned}
\|a b\|_{\widetilde{L}_{t}^{1}\left(\dot{B}_{2, r}^{-\frac{1}{2}}\right)} & \lesssim\|a\|_{\widetilde{L}_{t}^{4}\left(\dot{B}_{2, r}^{1}\right)}\|b\|_{\widetilde{L}_{t}^{\frac{4}{3}\left(\dot{B}_{2, r}^{0}\right)}}, \\
\|a b\|_{\widetilde{L}_{t}^{1}\left(\dot{B}_{2, r}^{-\frac{1}{2}}\right)} & \lesssim\|a\|_{\widetilde{L}_{t}^{\frac{4}{3}\left(\dot{B}_{2, r}^{1}\right)}}\|b\|_{\widetilde{L}_{t}^{4}\left(\dot{B}_{2, r}^{0}\right)} .
\end{aligned}
$$

Next, owing to Inequality (5.2) and interpolation, we have

$$
\begin{aligned}
& \left\|\left(\nabla \times v_{1}\right) \times \delta B\right\|_{\widetilde{L}_{t}^{1}\left(\dot{B}_{2, r}^{\frac{1}{2}}\right)}+\left\|v_{1} \cdot \nabla \delta B\right\|_{\widetilde{L}_{t}^{1}\left(\dot{B}_{2, r}^{\frac{1}{2}}\right)}+\left\|\delta v \cdot \nabla B_{2}\right\|_{\widetilde{L}_{t}^{1}\left(\dot{B}_{2, r}^{\frac{1}{2}}\right)} \\
& \lesssim\|\delta B\|_{\widetilde{L}_{t}^{4}\left(\dot{B}_{2, r}^{1},\right.}\left\|\nabla v_{1}\right\|_{\widetilde{L}_{t}^{\frac{4}{3}\left(\dot{B}_{2, r}^{1}\right)}}+\|\nabla \delta B\|_{\widetilde{L}_{t}^{\frac{4}{3}\left(\dot{B}_{2, r}^{1}\right)}}\left\|v_{1}\right\|_{\widetilde{L}_{t}^{4}\left(\dot{B}_{2, r}^{1}\right)} \\
& +\|\delta v\|_{\widetilde{L}_{t}^{\frac{4}{3}\left(\dot{B}_{2, r}^{1}\right)}}\left\|\nabla B_{2}\right\|_{\widetilde{L}_{t}^{4}\left(\dot{B}_{2, r}^{1}\right)} \\
& \lesssim\left(\left\|\left(u_{2}, v_{2}, v_{1}\right)\right\|_{\widetilde{L}_{t}^{4}\left(\dot{B}_{2, r}^{1}\right)}+\left\|v_{1}\right\|_{\widetilde{L}_{t}^{\frac{4}{3}\left(\dot{B}_{2, r}^{2}\right)}}\right)\|(\delta u, \delta v)\|_{F_{2, r}(t)} .
\end{aligned}
$$

Finally, applying (A.9) with $\rho=4, s=1 / 2$ and using embeddings $\dot{B}_{2, r}^{0} \hookrightarrow \dot{B}_{\infty}^{-\frac{3}{2}}$ and $\dot{B}_{2, r}^{1} \hookrightarrow \dot{B}_{\infty, \infty}^{-\frac{1}{2}}$ yields

$$
\left\|2^{\frac{j}{2}}\right\|\left[\dot{\Delta}_{j}, B_{2} \times\right](\nabla \times \delta v)\left\|_{L_{t}^{1}\left(L^{2}\right)}\right\|_{\ell^{r}(\mathbb{Z})} \lesssim\left\|\nabla B_{2}\right\|_{L_{t}^{4}\left(\dot{B}_{2, r}^{1}\right)}\|\nabla \times \delta v\|_{\widetilde{L}_{t}^{\frac{4}{3}\left(\dot{B}_{2, r}^{0}\right)}} .
$$

Thus, one can conclude that

$$
\|(\delta u, \delta B, \delta v)\|_{F_{2, r}(t)} \leq Y(t)\|(\delta u, \delta B, \delta v)\|_{F_{2, r}(t)}
$$

with $Y(t):=\sum_{i=1,2}\left\|\left(u_{i}, B_{i}, v_{i}\right)\right\|_{\widetilde{L}_{t}^{4}\left(\dot{B}_{2, r}^{1}\right)}+\left\|v_{1}\right\|_{\widetilde{L}_{t}^{\frac{4}{3}\left(\dot{B}_{2, r}^{2}\right)}}$.

Now, Lebesgue dominated convergence theorem ensures that $Y$ is a continuous nondecreasing function vanishing at zero. Hence $(\delta u, \delta B, \delta v) \equiv 0$ in $\widetilde{L}_{t}^{\infty}\left(\dot{B}_{2, r}^{-\frac{1}{2}}\right) \cap$ $\widetilde{L}_{t}^{1}\left(\dot{B}_{2, r}^{\frac{3}{2}}\right)$ for small enough $t$. Combining with a standard connectivity argument allows to conclude that $(\delta u, \delta B, \delta v) \equiv 0$ on $\mathbb{R}^{+}$. This completes the proof of the theorem in the small data case. 
Let us briefly explain how the above arguments have to be modified so as to handle the case where only $v_{0}$ is small. Note that no smallness condition is needed whatsoever in the proof of uniqueness. As regards the existence part, we split $u$ and $B($ not $v)$ into $u=u^{L}+\widetilde{u}$ and $B=B^{L}+\widetilde{B}$ and repeat the proof of Proposition 5.1 on the system fulfilled by $(\widetilde{u}, \widetilde{B}, v)$ rather than (2.14). Instead of (5.4), we get

$$
\|(\widetilde{u}, \widetilde{B}, v)\|_{E_{2, r}(t)} \lesssim\left\|v_{0}\right\|_{\dot{B}_{2, r}^{\frac{1}{2}}}+\|(u, B, v)\|_{\widetilde{L}_{t}^{\frac{4}{3}\left(\dot{B}_{2, r}^{2}\right)}}\|(u, B, v)\|_{\widetilde{L}_{t}^{4}\left(\dot{B}_{2, r}^{1}\right)}
$$

from which we deduce that

$$
\begin{aligned}
\|(\widetilde{u}, \widetilde{B}, v)\|_{E_{2, r}(t)} & \lesssim\left\|v_{0}\right\|_{\dot{B}_{2, r}^{\frac{1}{2}}}+\left\|\left(u^{L}, B^{L}\right)\right\|_{\widetilde{L}_{t}^{\frac{4}{3}}\left(\dot{B}_{2, r}^{2}\right)}\left\|\left(u^{L}, B^{L}\right)\right\|_{\widetilde{L}_{t}^{4}\left(\dot{B}_{2, r}^{1}\right)} \\
& +\left\|\left(u^{L}, B^{L}\right)\right\|_{\widetilde{L}_{t}^{\frac{4}{3}}\left(\dot{B}_{2, r}^{2}\right) \cap \widetilde{L}_{t}^{4}\left(\dot{B}_{2, r}^{1}\right)}\|(\widetilde{u}, \widetilde{B}, v)\|_{E_{2, r}(t)}+\|(\widetilde{u}, \widetilde{B}, v)\|_{E_{2, r}(t)}^{2} .
\end{aligned}
$$

Since, by dominated convergence theorem, we have

$$
\lim _{t \rightarrow 0}\left(\left\|\left(u^{L}, B^{L}\right)\right\|_{\widetilde{L}_{t}^{\frac{4}{3}}\left(\dot{B}_{2, r}^{2}\right)}+\left\|\left(u^{L}, B^{L}\right)\right\|_{\widetilde{L}_{t}^{4}\left(\dot{B}_{2, r}^{1}\right)}\right)=0,
$$

it is easy to see that if $\left\|v_{0}\right\|_{\dot{B}_{2, r}^{\frac{1}{2}}}$ is small enough, then one can get a control on $\|(\widetilde{u}, \widetilde{B}, v)\|_{E_{2, r}(t)}$ for small enough $t$. From this, repeating essentially the same arguments as in the small data case, one gets a local-in-time existence statement.

\section{Appendix A. Besov Spaces and commutator estimates}

Here, we briefly recall the definition of the Littlewood-Paley decomposition, define Besov spaces and list some properties that have been used repeatedly in the paper. For the reader's convenience, we also prove some nonlinear and commutator estimates. More details and proofs may be found in e.g. 3].

The Littlewood-Paley decomposition is a dyadic localization procedure in the frequency space for tempered distributions over $\mathbb{R}^{d}$. To define it, fix some nonincreasing smooth radial function $\chi$ on $\mathbb{R}^{d}$, supported in (say) $B(0,4 / 3)$ and with value 1 on $B(0,3 / 4)$, and set $\varphi(\xi):=\chi(\xi / 2)-\chi(\xi)$. Then, we have

$$
\forall \xi \in \mathbb{R}^{d}, \chi(\xi)+\sum_{j \geq 0} \varphi\left(2^{-j} \xi\right)=1 \quad \text { and } \quad \forall \xi \in \mathbb{R}^{d} \backslash\{0\}, \sum_{j \in \mathbb{Z}} \varphi\left(2^{-j} \xi\right)=1 .
$$

The homogeneous dyadic blocks $\dot{\Delta}_{j}$ and low-frequency cut-off operator $\dot{S}_{j}$ are defined for all $j \in \mathbb{Z}$ by

$$
\begin{aligned}
& \dot{\Delta}_{j} u:=\varphi\left(2^{-j} D\right) u=2^{j d} \int_{\mathbb{R}^{d}} h\left(2^{j} y\right) u(x-y) d y \quad \text { with } \quad h:=\mathcal{F}^{-1} \varphi, \\
& \dot{S}_{j} u:=\chi\left(2^{-j} D\right) u=2^{j d} \int_{\mathbb{R}^{d}} \tilde{h}\left(2^{j} y\right) u(x-y) d y \quad \text { with } \quad \tilde{h}:=\mathcal{F}^{-1} \chi .
\end{aligned}
$$

The following Littlewood-Paley decomposition of $u$ :

$$
u=\sum_{j \in \mathbb{Z}} \dot{\Delta}_{j} u
$$

holds true modulo polynomials for any tempered distribution $u$. In order to have an equality in the sense of tempered distributions, we consider only elements of the set $\mathcal{S}_{h}^{\prime}\left(\mathbb{R}^{d}\right)$ of tempered distributions $u$ such that

$$
\lim _{j \rightarrow-\infty}\left\|\dot{S}_{j} u\right\|_{L^{\infty}}=0 .
$$


Definition A.1. Let $s$ be a real number and $(p, r)$ be in $[1, \infty]^{2}$. The homogeneous Besov space $\dot{B}_{p, r}^{s}$ is the set of distributions $u$ in $\mathcal{S}_{h}^{\prime}$ such that

$$
\|u\|_{\dot{B}_{p, r}^{s}}:=\left\|2^{j s}\right\| \dot{\Delta}_{j} u\left\|_{L^{p}\left(\mathbb{R}^{d}\right)}\right\|_{\ell^{r}(\mathbb{Z})}<\infty .
$$

Proposition A.2. The following properties hold true:

(i) Derivatives: for all $s \in \mathbb{R}$ and $1 \leq p, r \leq \infty$, we have

$$
\sup _{|\alpha|=k}\left\|\partial^{\alpha} u\right\|_{\dot{B}_{p, r}^{s}} \sim\|u\|_{\dot{B}_{p, r}^{s+k}}
$$

(ii) Embedding: we have the following continuous embedding

$$
\dot{B}_{p, r}^{s} \hookrightarrow \dot{B}_{\tilde{p}, \tilde{r}}^{s-d\left(\frac{1}{p}-\frac{1}{\tilde{p}}\right)} \quad \text { whenever } \tilde{p} \geq p \quad \text { and } \quad \tilde{r} \geq r,
$$

and the space $\dot{B}_{p, 1}^{\frac{d}{p}}$ is embedded in the set of bounded continuous functions.

(iii) Real interpolation: for any $\theta \in(0,1)$ and $s<\tilde{s}$, we have

$$
\|u\|_{\dot{B}_{p, 1}^{\theta s+(1-\theta) \tilde{s}}} \lesssim\|u\|_{\dot{B}_{p, \infty}^{s}}^{\theta}\|u\|_{\dot{B}_{p, \infty}^{\tilde{s}}}^{1-\theta}
$$

(iv) Completeness: the space $\dot{B}_{p, r}^{s}$ is complete if (and only if) $(s, p, r)$ satisfies

$$
s<\frac{d}{p}, \quad \text { or } s=\frac{d}{p} \quad \text { and } \quad r=1 .
$$

(v) Density: the space $\mathcal{S}_{0}\left(\mathbb{R}^{d}\right)$ of Schwartz functions on $\mathbb{R}^{d}$ with Fourier transform supported away from the origin is dense in $\dot{B}_{p, r}^{s}$ whenever both $p$ and $r$ are finite.

(vi) Scaling invariance: for any $s \in \mathbb{R}$ and $(p, r) \in[1, \infty]^{2}$, there exists a constant $C$ such that for all positive $\lambda$ and $u \in \dot{B}_{p, r}^{s}$, we have

$$
C^{-1} \lambda^{s-\frac{d}{p}}\|u\|_{\dot{B}_{p, r}^{s}} \leq\|u(\lambda \cdot)\|_{\dot{B}_{p, r}^{s}} \leq C \lambda^{s-\frac{d}{p}}\|u\|_{\dot{B}_{p, r}^{s}} .
$$

(vii) Let $f$ be a smooth function on $\mathbb{R}^{d} \backslash\{0\}$ which is homogeneous of degree 0. Define $f(D)$ on $\mathcal{S}\left(\mathbb{R}^{d}\right)$ by

$$
\mathcal{F}(f(D) u)(\xi):=f(\xi) \mathcal{F} u(\xi),
$$

Then, for all exponents $(s, p, r)$, we have the estimate

$$
\|f(D) u\|_{\dot{B}_{p, r}^{s}} \lesssim\|u\|_{\dot{B}_{p, r}^{s}} .
$$

If in addition $f(D)$ extends to a map from $\mathcal{S}_{h}^{\prime}\left(\mathbb{R}^{d}\right)$ to itself and (A.1) is fulfilled, then $f(D)$ is continuous from $\dot{B}_{p, r}^{s}$ to $\dot{B}_{p, r}^{s}$.

(viii) Operator $\operatorname{curl}^{-1}$ maps $\dot{B}_{p, 1}^{s-1}$ to $\dot{B}_{p, 1}^{s}$ if $1 \leq p<\infty$ and $s \leq d / p$.

Proof. We only prove the last item as it is fundamental in our analysis. Owing to the definition in (1.10), it is obvious that $\operatorname{curl}^{-1}$ maps $\mathcal{S}_{0}\left(\mathbb{R}^{d}\right)$ to itself, and homogeneity of degree -1 implies that we have for all $u$ in $\mathcal{S}_{0}\left(\mathbb{R}^{d}\right)$ :

$$
\left\|\operatorname{curl}^{-1} u\right\|_{\dot{B}_{p, 1}^{s}} \lesssim\|u\|_{\dot{B}_{p, 1}^{s-1}} .
$$

As $\mathcal{S}_{0}\left(\mathbb{R}^{d}\right)$ is dense in $\dot{B}_{p, 1}^{s-1}$ and since the space $\dot{B}_{p, 1}^{s}$ is complete (owing to $s \leq d / p$ ), we get the result. 
A great deal of our analysis relies on regularity estimates for the heat equation:

$$
\left\{\begin{array}{l}
\partial_{t} u-\Delta u=f, \\
\left.u\right|_{t=0}=u_{0} .
\end{array}\right.
$$

It is classical that for all $u_{0} \in \mathcal{S}^{\prime}\left(\mathbb{R}^{d}\right)$ and $f \in L_{\text {loc }}^{1}\left(\mathbb{R}^{+} ; \mathcal{S}^{\prime}\left(\mathbb{R}^{d}\right)\right)$, equation $(H)$ has a unique tempered distribution solution, given by the following Duhamel formula:

$$
u(t)=e^{t \Delta} u_{0}+\int_{0}^{t} e^{(t-\tau) \Delta} f(\tau) d \tau, \quad t \geq 0 .
$$

Above, $\left(e^{t \Delta}\right)_{t \geq 0}$ stands for the heat semi-group. It is defined on $\mathcal{S}\left(\mathbb{R}^{d}\right)$ by

$$
\mathcal{F}\left(e^{t \Delta} z\right)(\xi):=e^{-t|\xi|^{2}} \widehat{z}(\xi),
$$

and is extended to the set of tempered distributions by duality.

As observed by Chemin in [10], the following spaces are suitable for describing the maximal regularity properties of the heat equation.

Definition A.3. For $T>0, s \in \mathbb{R}, 1 \leq \rho \leq \infty$, we set

$$
\|u\|_{\widetilde{L}_{T}^{\rho}\left(\dot{B}_{p, r}^{s}\right)}:=\left\|2^{j s}\right\| \dot{\Delta}_{j} u\left\|_{L_{T}^{\rho}\left(L^{p}\right)}\right\|_{\ell^{r}(\mathbb{Z})} .
$$

We define the space $\widetilde{L}_{T}^{\rho}\left(\dot{B}_{p, r}^{s}\right)$ to be the set of tempered distribution u on $(0, T) \times \mathbb{R}^{d}$ such that $\lim _{j \rightarrow-\infty}\left\|\dot{S}_{j} u(t)\right\|_{L^{\infty}}=0$ a.e. in $(0, T)$, and $\|u\|_{\tilde{L}_{T}^{\rho}\left(\dot{B}_{p, r}^{s}\right)}<\infty$. The space $\widetilde{L}_{T}^{\rho}\left(\dot{B}_{p, r}^{s}\right) \cap \mathcal{C}\left([0, T] ; \dot{B}_{p, r}^{s}\right)$ is denoted by $\widetilde{\mathcal{C}}_{T}\left(\dot{B}_{p, r}^{s}\right)$. In the case $T=+\infty$, one denotes the corresponding space and norm by $\widetilde{L}^{\rho}\left(\dot{B}_{p, r}^{s}\right)$ and $\|\cdot\|_{\widetilde{L}^{\rho}\left(\dot{B}_{p, r}^{s}\right)}$, respectively.

The above spaces or norms may be compared to more classical ones according to Minkowski's inequality:

$$
\|u\|_{\tilde{L}_{T}^{\rho}\left(\dot{B}_{p, r}^{s}\right)} \leq\|u\|_{L_{T}^{\rho}\left(\dot{B}_{p, r}^{s}\right)} \text { if } r \geq \rho \quad \text { and } \quad\|u\|_{\widetilde{L}_{T}^{\rho}\left(\dot{B}_{p, r}^{s}\right)} \geq\|u\|_{L_{T}^{\rho}\left(\dot{B}_{p, r}^{s}\right)} \text { if } r \leq \rho .
$$

The following fundamental result has been proved in [10].

Proposition A.4. Let $T>0, s \in \mathbb{R}$ and $1 \leq \rho, p, r \leq \infty$. Assume that $u_{0} \in \dot{B}_{p, r}^{s}$ and $f \in \widetilde{L}_{T}^{\rho}\left(\dot{B}_{p, r}^{s-2+\frac{2}{\rho}}\right)$. Then, $(H)$ has a unique solution $u$ in $\widetilde{L}_{T}^{\rho}\left(\dot{B}_{p, r}^{s+\frac{2}{\rho}}\right) \cap \widetilde{L}_{T}^{\infty}\left(\dot{B}_{p, r}^{s}\right)$ and there exists a constant $C$ depending only ond and such that for all $\rho_{1} \in[\rho, \infty]$, we have

$$
\|u\|_{\widetilde{L}_{T}^{\rho_{1}\left(\dot{B}_{p, r}^{\left.s+\frac{2}{\rho_{1}}\right)}\right.}} \leq C\left(\left\|u_{0}\right\|_{\dot{B}_{p, r}^{s}}+\|f\|_{\widetilde{L}_{T}^{\rho}\left(\dot{B}_{p, r}^{s-2+\frac{2}{\rho}}\right)}\right) .
$$

Furthermore, if $r$ is finite, then $u$ belongs to $\mathcal{C}\left([0, T] ; \dot{B}_{p, r}^{s}\right)$.

Let us now recall a few nonlinear estimates in Besov spaces, that we used in the paper. They all may be easily proved by using the following so-called Bony decomposition (from [6]) for the (formal) product of two distributions $u$ and $v$ :

$$
u v=T_{u} v+T_{v} u+R(u, v) .
$$

Above, $T$ designates the paraproduct bilinear operator defined by

$$
T_{u} v:=\sum_{j} \dot{S}_{j-1} u \dot{\Delta}_{j} v, \quad T_{v} u:=\sum_{j} \dot{S}_{j-1} v \dot{\Delta}_{j} u
$$


and $R$ stands for the remainder operator given by

$$
R(u, v):=\sum_{j} \sum_{\left|j^{\prime}-j\right| \leq 1} \dot{\Delta}_{j} u \dot{\Delta}_{j^{\prime}} v .
$$

The following properties of the paraproduct and remainder operators are classical:

Proposition A.5. For any $(s, p, r) \in \mathbb{R} \times[1, \infty]^{2}$ and $t<0$, there exists a constant $C$ such that

$$
\left\|T_{u} v\right\|_{\dot{B}_{p, r}^{s}} \leq C\|u\|_{L^{\infty}}\|v\|_{\dot{B}_{p, r}^{s}} \quad \text { and } \quad\left\|T_{u} v\right\|_{\dot{B}_{p, r}^{s+t}} \leq C\|u\|_{\dot{B}_{\infty, \infty}^{t}}\|v\|_{\dot{B}_{p, r}^{s}} .
$$

For any $\left(s_{1}, p_{1}, r_{1}\right)$ and $\left(s_{2}, p_{2}, r_{2}\right)$ in $\mathbb{R} \times[1, \infty]^{2}$ satisfying

$$
s_{1}+s_{2}>0, \quad \frac{1}{p}:=\frac{1}{p_{1}}+\frac{1}{p_{2}} \leq 1 \quad \text { and } \quad \frac{1}{r}:=\frac{1}{r_{1}}+\frac{1}{r_{2}} \leq 1,
$$

there exists a constant $C$ such that

$$
\|R(u, v)\|_{\dot{B}_{p, r}^{s_{1}+s_{2}}} \leq C\|u\|_{\dot{B}_{p_{1}, r_{1}}^{s_{1}}}\|v\|_{\dot{B}_{p_{2}, r_{2}}^{s_{2}}} .
$$

Combining the above proposition with the Bony decomposition allows to get a number of inequalities like, for instance:

- tame estimates: for any $s>0$ and $1 \leq p, r \leq \infty$,

$$
\|u v\|_{\dot{B}_{p, r}^{s}} \lesssim\|u\|_{L^{\infty}}\|v\|_{\dot{B}_{p, r}^{s}}+\|v\|_{L^{\infty}}\|u\|_{\dot{B}_{p, r}^{s}}
$$

- the following product estimate:

$$
\|u v\|_{\dot{B}_{p, 1}^{s_{1}+s_{2}-\frac{d}{p}}} \lesssim\|u\|_{\dot{B}_{p, 1}^{s_{1}}}\|v\|_{\dot{B}_{p, 1}^{s_{2}}}
$$

whenever $s_{1}, s_{2} \leq \frac{d}{p}$ satisfy $s_{1}+s_{2}>d \max \left(0, \frac{2}{p}-1\right)$;

- the following inequality (in the case $d=3$ and $\rho>2$ ) that has been used in the proof of (2.18):

$$
\|a b\|_{\dot{B}_{2,1}^{\frac{3}{2}}} \lesssim\|a\|_{\dot{B}_{\infty, \infty}^{\frac{2}{\rho}-1}}\|b\|_{\dot{B}_{2,1}^{\frac{5}{2}-\frac{2}{\rho}}}+\|b\|_{\dot{B}_{\infty, \infty}^{\frac{2}{\rho}-1}}\|a\|_{\dot{B}_{2,1}^{\frac{5}{2}-\frac{2}{\rho}}}
$$

Remark 4. Proposition A.5 and estimates like A.6 or A.7 may be adapted to the spaces $\widetilde{L}_{T}^{\rho}\left(\dot{B}_{p, r}^{s}\right)$. The general principle is that the time exponent behaves according to Hölder inequality. For example, we have

$$
\left\|T_{a} b\right\|_{\widetilde{L}_{t}^{1}\left(\dot{B}_{2, r}^{\frac{1}{2}}\right)}+\left\|T_{b} a\right\|_{\widetilde{L}_{t}^{1}\left(\dot{B}_{2, r}^{\frac{1}{2}}\right)}+\|R(a, b)\|_{\widetilde{L}_{t}^{1}\left(\dot{B}_{2, r}^{\frac{1}{2}}\right)} \lesssim\|a\|_{\widetilde{L}_{t}^{\frac{4}{3}}\left(\dot{B}_{\infty}^{-\frac{1}{2}}\right)}\|b\|_{\widetilde{L}_{t}^{4}\left(\dot{B}_{2, r}^{1}\right)} .
$$

Then, combining with embedding (in the case $d=3$ ) gives Inequality (5.2).

Similarly, Inequality (5.3) stems from

$$
\begin{aligned}
\left\|T_{a} b\right\|_{\widetilde{L}_{t}^{1}\left(\dot{B}_{2, r}^{\frac{3}{2}}\right)}+\left\|T_{b} a\right\|_{\widetilde{L}_{t}^{1}\left(\dot{B}_{2, r}^{\frac{3}{2}}\right)} & +\|R(a, b)\|_{\widetilde{L}_{t}^{1}\left(\dot{B}_{2, r}^{\frac{3}{2}}\right)} \\
& \lesssim\|a\|_{\widetilde{L}_{t}^{4}\left(\dot{B}_{\infty, \infty}^{-\frac{1}{2}}\right)}\|b\|_{\widetilde{L}_{t}^{\frac{4}{3}}\left(\dot{B}_{2, r}^{2}\right)}+\|b\|_{\widetilde{L}_{t}^{4}\left(\dot{B}_{\infty, \infty}^{-\frac{1}{2}}\right)}\|a\|_{\widetilde{L}_{t}^{\frac{4}{3}\left(\dot{B}_{2, r}^{2}\right)}} .
\end{aligned}
$$

\footnotetext{
${ }^{4}$ In particular, $\dot{B}_{p, 1}^{\frac{d}{p}}$ is an algebra for any $1 \leq p<\infty$.
} 
In order to prove Inequality (5.7), it suffices to use the fact that

$$
\begin{aligned}
\left\|T_{a} b\right\|_{\widetilde{L}_{t}^{1}\left(\dot{B}_{2, r}^{-\frac{1}{2}}\right)} & \lesssim\|a\|_{\widetilde{L}_{t}^{4}\left(\dot{B}_{\infty, \infty}^{-\frac{1}{2}}\right)}\|b\|_{\widetilde{L}_{t}^{\frac{4}{3}\left(\dot{B}_{2, r}^{0}\right)}}, \\
\left\|T_{b} a\right\|_{\widetilde{L}_{t}^{1}\left(\dot{B}_{2, r}^{-\frac{1}{2}}\right)} & \lesssim\|b\|_{\widetilde{L}_{t}^{\frac{4}{3}\left(\dot{B}_{\infty}^{-\frac{3}{2}}\right.}}\|a\|_{\widetilde{L}_{t}^{4}\left(\dot{B}_{2, r}^{1}\right)}, \\
\|R(a, b)\|_{\widetilde{L}_{t}^{1}\left(\dot{B}_{2, r}^{-\frac{1}{2}}\right)} & \lesssim\|a\|_{\widetilde{L}_{t}^{4}\left(\dot{B}_{2, r}^{1}\right)}\|b\|_{\widetilde{L}_{t}^{\frac{4}{3}\left(\dot{B}_{2, r}^{0}\right)}} .
\end{aligned}
$$

Proving Inequality (5.8) is similar.

We end this appendix with the proof of commutator estimates that were crucial in our analysis.

Proposition A.6. Let $s$ be in $(0, d / 2]$. Then, we have:

$$
\sum_{j \in \mathbb{Z}} 2^{j s}\left\|\left[\dot{\Delta}_{j}, b\right] a\right\|_{L^{2}} \lesssim\|\nabla b\|_{L^{\infty}}\|a\|_{\dot{B}_{2,1}^{s-1}}+\|a\|_{\dot{B}_{\infty, \infty}^{-1}}\|\nabla b\|_{\dot{B}_{2,1}^{s}} .
$$

Furthermore, for all $r \in[1, \infty]$ and $\rho \in(2, \infty]$, we have if we set $1 / \rho^{\prime}:=1-1 / \rho$,

$$
\begin{aligned}
\left\|2^{j s}\right\|\left[\dot{\Delta}_{j}, b\right] a\left\|_{L_{t}^{1}\left(L^{2}\right)}\right\|_{\ell^{r}(\mathbb{Z})} \lesssim\|\nabla b\|_{\widetilde{L}_{t}^{\rho}\left(\dot{B}_{\infty, \infty}^{\frac{2}{\rho}-1}\right)} & \|a\|_{\widetilde{L}_{t}^{\rho^{\prime}\left(\dot{B}_{2, r}^{s-\frac{2}{\rho}}\right)}} \\
& +\|\nabla b\|_{\widetilde{L}_{t}^{\rho^{\prime}\left(\dot{B}_{2, r}^{\left.s+1-\frac{2}{\rho}\right)}\right.}}\|a\|_{\widetilde{L}_{t}^{\rho}\left(\dot{B}_{\infty, \infty}^{\frac{2}{\rho}-2}\right)}
\end{aligned}
$$

Proof. Proving the two inequalities relies on the decomposition

$$
\left[\dot{\Delta}_{j}, b\right] a=\left[\dot{\Delta}_{j}, T_{b}\right] a+\dot{\Delta}_{j}\left(T_{a} b+R(a, b)\right)-\left(T_{\dot{\Delta}_{j} a} b+R\left(\dot{\Delta}_{j} a, b\right)\right) .
$$

For getting (A.8), we bound the first term of (A.10) as follows (use [3, Ineq. (2.58)]):

$$
\sum_{j \in \mathbb{Z}} 2^{j s}\left\|\left[\dot{\Delta}_{j}, T_{b}\right] a\right\|_{L^{2}} \lesssim\|\nabla b\|_{L^{\infty}}\|a\|_{\dot{B}_{2,1}^{s-1}}
$$

The next two terms of A.10 may be bounded by using the fact that the remainder and paraproduct operator map $\dot{B}_{\infty, \infty}^{-1} \times \dot{B}_{2,1}^{s+1}$ to $\dot{B}_{2,1}^{s}$. Finally, owing to the properties of localization of the Littlewood-Paley decomposition, we have

$$
T_{\dot{\Delta}_{j} a} b+R\left(\dot{\Delta}_{j} a, b\right)=\sum_{j^{\prime} \geq j-2} \dot{S}_{j^{\prime}+2} \dot{\Delta}_{j} a \dot{\Delta}_{j^{\prime}} b .
$$

From Bernstein inequality and $\left\|\dot{S}_{j^{\prime}+2} a\right\|_{L^{\infty}} \lesssim 2^{j^{\prime}}\|a\|_{\dot{B}_{\infty, \infty}^{-1}}$, we gather

$$
\begin{aligned}
\sum_{j} 2^{j s}\left\|T_{\dot{\Delta}_{j} a} b+R\left(\dot{\Delta}_{j} a, b\right)\right\|_{L^{2}} & \lesssim \sum_{j} \sum_{j^{\prime} \geq j-2} 2^{j s}\left\|\dot{S}_{j^{\prime}+2} a\right\|_{L^{\infty}}\left\|\dot{\Delta}_{j^{\prime}} b\right\|_{L^{2}} \\
& \lesssim\|a\|_{\dot{B}_{\infty, \infty}^{-1}} \sum_{j} \sum_{j^{\prime} \geq j-2} 2^{s\left(j-j^{\prime}\right)} 2^{j^{\prime} s}\left\|\nabla \dot{\Delta}_{j^{\prime}} b\right\|_{L^{2}} \\
& \lesssim\|a\|_{\dot{B}_{\infty, \infty}^{-1}}\|\nabla b\|_{\dot{B}_{2,1}^{s}} .
\end{aligned}
$$

To prove (A.9), we observe that owing to the localization properties of the Littlewood-Paley decomposition, the first term of A.10 may be decomposed into

$$
\left[\dot{\Delta}_{j}, T_{b}\right] a=\sum_{\left|j^{\prime}-j\right| \leq 4}\left[\dot{\Delta}_{j}, \dot{S}_{j^{\prime}-1} b\right] \dot{\Delta}_{j^{\prime}} a
$$

Now, according to [3, Lem. 2.97], we have

$$
\left\|\left[\dot{\Delta}_{j}, \dot{S}_{j^{\prime}-1} b\right] \dot{\Delta}_{j^{\prime}} a\right\|_{L^{2}} \lesssim 2^{-j}\left\|\nabla \dot{S}_{j^{\prime}-1} b\right\|_{L^{\infty}}\left\|\dot{\Delta}_{j^{\prime}} a\right\|_{L^{2}}
$$


and, since $\frac{2}{\rho}-1<0$,

$$
\left\|\nabla S_{j^{\prime}-1} b\right\|_{L_{t}^{\rho}\left(L^{\infty}\right)} \lesssim 2^{j^{\prime}\left(1-\frac{2}{\rho}\right)}\|\nabla b\|_{\widetilde{L}_{t}^{\rho}\left(\dot{B}_{\infty, \infty}^{\frac{2}{\rho}-1}\right)} .
$$

Hence, for all $\left(j, j^{\prime}\right) \in \mathbb{Z}^{2}$ such that $\left|j-j^{\prime}\right| \leq 4$,

$$
2^{j s}\left\|\left[\dot{\Delta}_{j}, \dot{S}_{j^{\prime}-1} b\right] \dot{\Delta}_{j^{\prime}} a\right\|_{L_{t}^{1}\left(L^{2}\right)} \lesssim 2^{j s} 2^{-\frac{2}{\rho} j^{\prime}}\left\|\dot{\Delta}_{j^{\prime}} a\right\|_{L_{t}^{\rho^{\prime}\left(L^{2}\right)}}\|\nabla b\|_{\widetilde{L}_{t}^{\rho}\left(\dot{B}_{\infty, \infty}^{\frac{2}{\rho}-1}\right)} .
$$

Therefore, summing up on $j^{\prime} \in\{j-4, j+4\}$, then taking the $\ell^{r}(\mathbb{Z})$ norm,

$$
\left\|2^{j s}\right\|\left[\dot{\Delta}_{j}, T_{b}\right] a\left\|_{L_{t}^{1}\left(L^{2}\right)}\right\|_{\ell^{r}} \lesssim\|\nabla b\|_{\widetilde{L}_{t}^{\rho}\left(\dot{B}_{\infty, \infty}^{\frac{2}{\rho}-1}\right)}\|a\|_{\widetilde{L}_{t}^{\rho^{\prime}\left(\dot{B}_{2, r}^{s-\frac{2}{\rho}}\right)}} .
$$

The next two terms may be bounded according to Proposition A.5 and Remark 4

$$
\left\|2^{j s}\right\| \dot{\Delta}_{j} T_{a} b\left\|_{L_{t}^{1}\left(L^{2}\right)}\right\| \ell_{\ell^{r}}+\left\|2^{j s}\right\| \dot{\Delta}_{j} R(a, b)\left\|_{L_{t}^{1}\left(L^{2}\right)}\right\|_{\ell^{r}} \lesssim\|a\|_{\widetilde{L}_{t}^{\rho}\left(\dot{B}_{\infty, \infty}^{\frac{2}{\rho}-2}\right)}\|\nabla b\|_{\widetilde{L}_{t}^{\rho^{\prime}\left(\dot{B}_{2, r}^{s+1-\frac{2}{\rho}}\right)}}
$$

Finally, use (A.11) and the fact that

$$
\left\|\dot{S}_{j^{\prime}+2} a\right\|_{L_{t}^{\rho}\left(L^{\infty}\right)} \lesssim 2^{\left(2-\frac{2}{\rho}\right) j^{\prime}}\|a\|_{\widetilde{L}_{t}^{\rho}\left(\dot{B}_{\infty, \infty}^{2}\right)}
$$

to get

$$
\begin{aligned}
2^{j s}\left\|T_{\dot{\Delta}_{j} a} b+R\left(\dot{\Delta}_{j} a, b\right)\right\|_{L_{t}^{1}\left(L^{2}\right)} & \lesssim \sum_{j^{\prime} \geq j-2} 2^{j s}\left\|\dot{S}_{j^{\prime}+2} a\right\|_{L_{t}^{\rho}\left(L^{\infty}\right)}\left\|\dot{\Delta}_{j^{\prime}} b\right\|_{L_{t}^{\rho^{\prime}}\left(L^{2}\right)} \\
& \lesssim\|a\|_{\widetilde{L}_{t}^{\rho}\left(\dot{B}_{\infty, \infty}^{\frac{2}{\rho}-2}\right)} \sum_{j^{\prime} \geq j-2} 2^{s\left(j-j^{\prime}\right)} 2^{\left(s+2-\frac{2}{\rho}\right) j^{\prime}}\left\|\dot{\Delta}_{j^{\prime}} b\right\|_{L_{t}^{\rho^{\prime}\left(L^{2}\right)}} .
\end{aligned}
$$

Taking the $\ell^{r}(\mathbb{Z})$ norm of both sides and using a convolution inequality for series (remember that $s>0$ ), we end up with

$$
\left\|2^{j s}\right\| T_{\dot{\Delta}_{j} a} b+R\left(\dot{\Delta}_{j} a, b\right)\left\|_{L_{t}^{1}\left(L^{2}\right)}\right\|_{\ell^{r}(\mathbb{Z})} \lesssim\|a\|_{\widetilde{L}_{t}^{\rho}\left(\dot{B}_{\infty, \infty}^{\frac{2}{\rho}-2}\right)}\|\nabla b\|_{\widetilde{L}_{t}^{\rho^{\prime}\left(\dot{B}_{2, r}^{s+1-\frac{2}{\rho}}\right)}} .
$$

This completes the proof of Inequality (A.9).

\section{REFERENCES}

[1] M. Acheritogaray, P. Degond, A. Frouvelle and J.-G. Liu, Kinetic formulation and global existence for the Hall-Magneto-hydrodynamics system, Kinet. Relat. Models, 4 (2011) 901-918.

[2] B. Ahmad, T. Hayat, F. He and Y. Zhou, On regularity criteria for the 3D Hall-MHD equations in terms of the velocity, Nonlinear Anal. Real World Appl., 32 (2016) 35-51.

[3] H. Bahouri, J.-Y. Chemin and R. Danchin, Fourier Analysis and Nonlinear Partial Differential Equations, 343, Grundlehren der Mathematischen Wissenschaften, Springer 2011.

[4] S.A. Balbus and C. Terquem, Linear analysis of the Hall effect in protostellar disks, Astrophys. J., 552 (2001) 235-247.

[5] M. Benvenutti and L. Ferreira, Existence and stability of global large strong solutions for the Hall-MHD system, Differential Integral Equations, 29 (2016) 977-1000.

[6] J. M. Bony, Calcul symbolique et propagation des singularités pour les équations aux dérivées partielles non linéaires, Ann. Sci. École Norm. Sup., 14(4) (1986) 209-246.

[7] M. Cannone, Ondelettes, paraproduits et Navier-Stokes, Diderot Editeur, Paris, 1995.

[8] D. Chae, P. Degond, and J.-G. Liu, Well-posedness for Hall-magnetohydrodynamics, Ann. IHP, Analyse non linéaire, 31 (2014) 555-565.

[9] D. Chae and J. Lee, On the blow-up criterion and small data global existence for the Hallmagnetohydrodynamics, J. Differential Equations, 256 (2014) 3835-3858.

[10] J.-Y. Chemin, Théorèmes d'unicité pour le système de Navier-Stokes tridimensionnel, Journal d'Analyse Mathématique, 77 (1999) 25-50. 
[11] N. Duan, Global well-posedness and analyticity of solutions to three-dimensional Hall-MHD equations, J. Math. Anal. Appl., 463 (2018) 506-516.

[12] E. Dumas And F. Sueur, On the weak solutions to the Maxwell-Landau-Lifshitz equations and to the Hall-Magneto-Hydrodynamic equations, Com. Math. Phys., 330 (2014) 1179-1225.

[13] T.G. Forbes, Magnetic reconnection in solar flares, Geophys. Astrophys. Fluid Dyn., 62 (1991) 15-36.

[14] J.D. HubA, Hall Magnetohydrodynamics - A Tutorial. In: Büchner J., Scholer M., Dum C.T. (eds) Space Plasma Simulation. Lecture Notes in Physics, 615. Springer, 2003.

[15] J. Li, Y. YU AND W. ZHU, A class large solution of the 3D Hall-magnetohydrodynamic equations, arXiv:1903.02299.

[16] C. Miao And B. Yuan, On the well-posedness of the Cauchy problem for an MHD system in Besov spaces, Mathematical Methods in the Applied Sciences, 32 (2009) 53-76.

[17] P.D. Mininni, Hall-MHD dynamos and turbulence, http://www.ens-lyon.fr/PHYSIQUE/ Equipe2/LesHouches11/Talks_files/Mininni_Houches2011.pdf.

[18] D.A. Shalybkov And V.A. Urpin, The Hall effect and the decay of magnetic fields, Astron. Astrophys., 321 (1997) 685-690.

[19] M. WARdLe, Star formation and the Hall effect, Astrophys. Space Sci. 292 (2004) 317-323.

[20] X. WU, Y. YU AND Y. TANG, Well-posedness for the Incompressible Hall-MHD Equations in Low Regularity Spaces, Mediterr. J. Math. 15(2) (2018) article 48.

[21] R. Wan And Y. Zhou, On global existence, energy decay and blow-up criteria for the HallMHD system, J. Differ. Equ., 259 (2015) 5982-6008.

[22] R. WAN AND Y. ZHOU, Low regularity well-posedness for the 3D generalized Hall-MHD system, Acta Appl. Math. 147 (2017) 95-111.

[23] R. WAn And Y. Zhou, Global well-posedness, BKM blow-up criteria and zero $h$ limit for the 3D incompressible Hall-MHD equations, J. of Diff. Equations 267 (2019) 3724-3747.

[24] R. WAN AND Y. ZHOU, Global well-posedness for the 3D incompressible Hall-magnetohydrodynamic equations with Fujita-Kato type initial data, J. Math. Fluid Mech., 21(1) (2019), Art. 5, 16 pp.

[25] Z. YE, Regularity criteria and small data global existence to the generalized viscous Hallmagnetohydrodynamics, Comp. and Math. with Applications, 70 (2015) 2137-2154.

Université Paris-Est Créteil, LAMA UMR 8050, 61 avenue du GÉnÉral de Gaulle, 94010 Créteil et Sorbonne Université, LJLL UMR 7598, 4 Place Jussieu, 75005 Paris

E-mail address: raphael.danchin@u-pec.fr

Université Paris-Est Créteil, LAMA UMR 8050, 61 avenue du GÉnÉral de Gaulle, 94010 Créteil

E-mail address: jin.tan@u-pec.fr 